\title{
EFFECTS OF LARGE-SCALE FREE STREAM TURBULENCE ON A TURBULENT BOUNDARY LAYER
}

\author{
A Thesis \\ Presented to the Faculty of the Graduate School \\ of Cornell University \\ In Partial Fulfillment of the Requirements for the Degree of \\ Master of Science
}

by

Nicole Susanne Sharp

May 2009 
(C) 2009 Nicole Susanne Sharp 


\begin{abstract}
Results of a wind tunnel experiment in which there are systematic variations of free stream turbulence above a flat plate boundary layer are presented. Upstream of the plate, an active grid generates free stream turbulence varying in intensity from $0.25 \%$ to $10.5 \%$. The momentum thickness Reynolds number of the boundary layer varies from 550 to nearly 3,000 . In all cases, the ratio of the free stream turbulence length scale to the boundary layer depth is greater than unity. Hot wire measurements show that, at high turbulence intensities, the effects of the free stream turbulence extend deep into the boundary layer, affecting the wall stress as well as the small-scale (derivative) statistics. Energy spectra show a double peak. At very low free stream turbulence intensities these peaks are associated with the inner and outer scales of the turbulent boundary layer, but at high turbulence intensities the free stream energy peak dominates over the boundary layer's outer scale. The results are contrasted with recent studies of turbulent boundary layers without free stream turbulence.
\end{abstract}




\section{BIOGRAPHICAL SKETCH}

Nicole Sharp graduated magna cum laude from Case Western Reserve University in May 2006 with a Bachelor of Science in Engineering in aerospace engineering. While at Case Western, she was awarded a fellowship from the Support of Undergraduate Research and Creative Endeavors (SOURCE) office to pursue interdisciplinary research on the odor-sensing abilities of the Manduca sexta moth. For this work, she received second place in the Engineering and Computer Science category of the 2006 SOURCE Undergraduate Symposium and Poster Session. She began her tenure at Cornell University in August 2006 and is currently pursuing doctoral research at Texas A\&M University. 
To Mom and Dad, who always encouraged me to dream big and who taught me the strength of character needed to pursue those dreams. 


\section{ACKNOWLEDGMENTS}

First and foremost, I would like to thank Stephanie Neuscamman, both for her help in every step of this project and for her constant support and friendship. I'd also like to thank other labmates who have helped along the way: Juan Isaza, who was always willing to donate his time and car toward moving equipment; Erika Johnson, who was always happy to lend a hand; Doug Kutz, who spent a summer helping with tasks both large and small while the experiments were going on; Sergiy Gerashchenko, who designed and fabricated the flat-plate apparatus used in the experiments; and Sathyanarayana Ayyalasomayajula, who taught me the tools of the trade. Thanks are also due to my committee members, Profs. Zellman Warhaft and Lance Collins, for their support, guidance, and patience with me along the way. This work was supported by funds from the U.S. National Science Foundation, and I gratefully acknowledge their contribution.

On a personal level, I'd like to thank my parents and sister; without their love and encouragement, I would never have made it this far. And, finally, I thank Joseph Shoer, who was always there to congratulate, to encourage, to comfort, and to motivate me in spite of myself. My sincerest thanks to you all.

- Nicole S. Sharp

May 2009 
TABLE OF CONTENTS

Biographical Sketch iii

Dedication $\quad$ iv

Acknowledgements $\quad$ V

List of Figures vii

List of Tables viii

Introduction 1

$\begin{array}{ll}\text { Apparatus } & 4\end{array}$

$\begin{array}{ll}\text { Results } & 6\end{array}$

$\begin{array}{ll}\text { Conclusions } & 37\end{array}$

$\begin{array}{ll}\text { References } & 40\end{array}$ 


\section{LIST OF FIGURES}

Figure 1: Schematic of experimental apparatus $\quad 4$

$\begin{array}{ll}\text { Figure 2: Mean velocity profiles } & 7\end{array}$

$\begin{array}{lr}\text { Figure 3: Mean velocity defect profiles } & 8\end{array}$

$\begin{array}{lr}\text { Figure 4: Turbulence intensity profiles } & 10\end{array}$

Figure 5: Velocity variance and covariance profiles 11

Figure 6: Cross-correlation coefficient profiles $\quad 14$

$\begin{array}{ll}\text { Figure 7: Anisotropy profiles } & 15\end{array}$

Figure 8: Free stream and near-wall PDFs 16

$\begin{array}{lr}\text { Figure 9: Skewness profiles } & 20\end{array}$

$\begin{array}{ll}\text { Figure 10: Kurtosis profiles } & 21\end{array}$

Figure 11: Derivative skewness profiles $\quad 22$

$\begin{array}{lr}\text { Figure 12: Derivative kurtosis profiles } & 23\end{array}$

$\begin{array}{ll}\text { Figure 13: Power spectra } & 25\end{array}$

Figure 14: Compensated power spectra 26

Figure 15: Normalized energy spectra at $y / \delta=0.05-0.06 \quad 28$

Figure 16: Evolution of normalized energy spectra for $R e_{\lambda 0}=260$

Figure 17: Three-dimensional evolution of normalized energy spectra 32 


\section{LIST OF TABLES}

Table 1: Experimental flow parameters 


\section{INTRODUCTION}

Above industrial and naturally occurring boundary layers there is invariably free stream turbulence (FST). Examples include nocturnal jets or other highly turbulent flows that may occur at the top of the atmospheric boundary layer [1] or a marine atmospheric boundary layer that may override a boundary layer forming above land. In turbomachinery and heat exchangers, there is usually free stream turbulence [2]. The FST may affect stress conditions near the surface, thereby impacting the performance of wind turbines [3] and other structures located in the boundary layer. It causes greater irregularity at the interface of the boundary layer and adds a new length scale, compounding an already complex problem. The free stream turbulence may have a length scale that is small, comparable to, or larger than that of the boundary layer outer length scale, and the r.m.s. of its velocity fluctuations may vary from being significantly smaller than the friction velocity, $u_{\tau}$, to much larger than it, yielding at least nine possible forcing states for the FST. Some of these states are discussed by Hunt et al. (1998). [4] One of the most interesting states occurs when both the free stream length scale, $L$, and the turbulence intensity, $\left\langle u^{2}\right\rangle^{1 / 2} / U\left(\right.$ where $\left\langle u^{2}\right\rangle^{1 / 2}$ is the longitudinal r.m.s. velocity and $U$ is the local mean velocity), are considerably larger than that of the boundary layer. For this case, there is penetration of the FST deep into the boundary layer, resulting in an increase in the friction velocity, and there is little or no velocity defect in the outer part of the boundary layer. It is a case of considerable practical importance and is the focus of this paper.

There is a significant literature on the effects of FST on a turbulent boundary layer [5-14]. The FST affects the wall stress, the turbulent energy budget, the velocity spectra, and the velocity probability distribution. For very high FST, the effects are felt as close to the wall as $y^{+} \sim 15$ [10]. Here $y^{+}$is defined as $y u_{\tau} / v$ where $y$ is the 
distance from the wall and $v$ is the kinematic viscosity, taken to be $1.5 \times 10^{-5} \mathrm{~m}^{2} \mathrm{~s}^{-1}$ in air. Thus the large eddies from the free stream can perturb the smaller, near-wall structures. Recently, Hutchins and Marusic [15] have emphasized the important interaction between the outer scale and the inner (viscous) scale in a boundary layer without FST. They show that the energy of the outer scale grows as the boundary layer Reynolds number increases and that these energetic eddies modulate the small scales of the boundary layer, causing changes in small-scale structure and drag. For intense FST with scales comparable to those of the outer scale of the boundary layer, this effect is bound to be amplified. Indeed, we will show that, for the high FST cases studied here, the energy of the FST masks that of the boundary layer's outer scale. Thus the FST, apart from its practical interest, may shed light on our fundamental understanding of the interactions between the large- and small-scales in the turbulent boundary layer.

Various methods have been used to produce FST turbulence in wind tunnel studies. The most traditional is to use a turbulence generating grid [7]. With this method, the maximum FST intensity is around 6\%. This intensity is not sufficient to see the effects of the FST deep inside the boundary layer. In order to increase the FST intensity, Thole and Bogard [10] used a turbulence generator consisting of a row of high-velocity jets entering the flow normal to and upstream of the boundary layer. They were able to obtain turbulence intensities of $20 \%$. In the present work, we generate the turbulence using an active grid [17-18] upstream of the boundary layer. This has the advantage of being a well-documented flow in its own right [17_-19].

For all of these methods, the FST is decaying. In order to have non-decaying turbulence in the free stream, a production method like inhomogeneity or shear is 
necessary [20]. In this situation, however, the problem is even more complex due to anisotropy in the free stream. In this paper, we will consider mildly anisotropic decaying free stream turbulence with r.m.s. values more than twice the friction velocity and free stream turbulence length scales approximately five times the boundary layer thickness, $\delta$, based on $99.5 \%$ of the mean free stream velocity. The maximum turbulence intensity will be around $10 \%$.

The work described here was motivated by the experiment of Gerashchenko et al. (2008) [21], which used a similar configuration to study the acceleration of inertial particles in a boundary layer with FST. In that paper, results of the boundary layer mean and variance velocity profiles were described. Here, using a different flow configuration, we document the boundary layer statistics, including higher order moments and spectra, measured using hotwire anemometry. We systematically vary the free stream turbulent Reynolds number ${ }^{1}, \operatorname{Re}_{\lambda 0}\left(\equiv\left\langle u^{2}\right\rangle^{1 / 2} \lambda / v\right.$, where $\lambda$, the Taylor micro-scale, defined as $\left.\left\langle u^{2}\right\rangle^{1 / 2} /\left\langle(d u / d x)^{2}\right\rangle^{1 / 2}\right)$; the free stream turbulence intensity; and the boundary layer Reynolds number, $\operatorname{Re}_{\theta}$, defined as $\operatorname{Re}_{\theta}=U_{0} \theta / v$, where $U_{0}$ is the mean velocity in the free stream and $\theta$, the momentum thickness, is defined as ${ }_{0} \int^{\infty}\left(U / U_{0}\right)\left(1-\left(U / U_{0}\right) d y\right.$. The results are compared with other experiments that have addressed this problem, notably the work of Thole and Bogard [10], as well as with a turbulent boundary layer without FST [15-16].

\footnotetext{
${ }^{1}$ In this paper, a + superscript (e.g. $y^{+}, u^{+}, \lambda^{+}$, etc.) indicates a quantity non-dimensionalized by $u_{\tau}$, the friction velocity, and $v$, the kinematic viscosity. Similarly, a subscript 0 (e.g. $\left.U_{0}\right)$ is used to indicate free stream values.
} 


\section{APPARATUS}

The experiments took place in a horizontal, open-circuit wind tunnel with a crosssection of $91.4 \times 91.4 \mathrm{~cm}^{2}$ and a $9.1 \mathrm{~m}$ long test section [22]. A smooth glass plate $3.3 \mathrm{~m} \times 0.67 \mathrm{~m} \times 0.012 \mathrm{~m}$, as used by Gerashchenko et al. (2008) in a different wind tunnel [21], placed $0.34 \mathrm{~m}$ above the floor of the tunnel provided the surface for the formation of a boundary layer. Figure 1 shows a schematic of the experimental apparatus, including relevant dimensions. No special techniques were used to trip the boundary layer at the plate's edge as it was assumed that the FST would have a stronger effect on the boundary layer than a tripping mechanism would. Due to the fixed height of the wind tunnel, there was a slight favorable pressure gradient in the $x$ direction in the absence of FST. In the presence of FST, however, no pressuregradient was found.

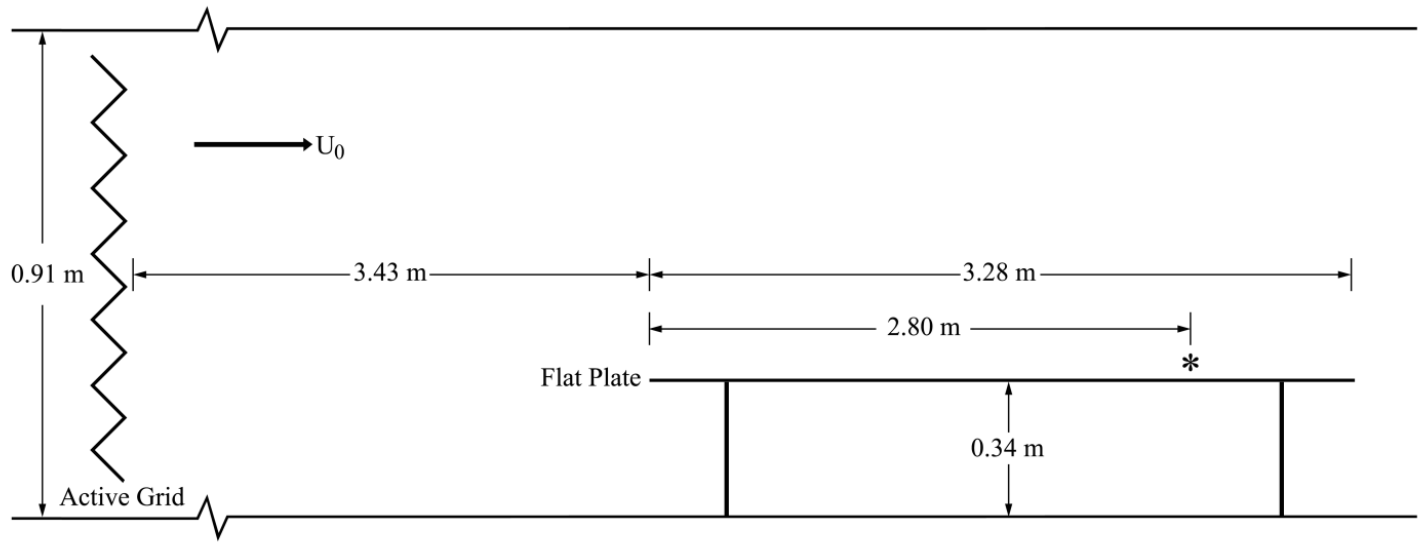

Figure 1: Schematic of wind tunnel test section with placement of flat-plate. The asterisk marks the location of the measurement station.

An active turbulence-generating grid (hereafter referred to as an active grid) placed at the beginning of the test section created nearly-isotropic turbulence in the free stream. The grid consisted of bars with attached triangular agitator wings that flip and rotate randomly [17-18]. The active grid was used in two ways during the experiments. To achieve high levels of FST intensity, the active grid operated in its normal, random 
mode. The active grid was also used in a passive mode by aligning the bars of the grid such that the agitator wings were in the plane of the mean flow. For this case, the FST was significantly reduced. Some experiments were done without any grid to achieve a nearly quiescent free stream. The mesh spacing for the grid is $M=11.4 \mathrm{~cm}$. The glass plate used to generate the boundary layer was located $30 M(3.43 \mathrm{~m})$ downstream of the active grid to allow for the development of the grid-generated turbulence before it arrived at the plate.

Constant temperature hotwire anemometry provided the data used to characterize the flow. A boundary layer X-wire from TSI (Model 1243-T1.5) with tungsten wires 3.05 $\mu \mathrm{m}$ in diameter measured mean velocities and velocity fluctuations in both the streamwise and vertical (plate-normal) directions. The ratio of the active length to the diameter, $l / d$, of the wires was approximately 200 . The hotwires were connected to Disa 55M01 constant-temperature bridges, and the signals from these passed through high-pass $(0.01 \mathrm{~Hz})$ and low-pass (between 1,000 and 10,000 Hz) filters to reduce large-scale disturbances and high-frequency noise prior to digitization. 


\section{RESULTS}

Data were recorded for eight different free stream conditions, ranging from nearly laminar to highly turbulent, so that the increasing effects of free stream turbulence could be explored. The parameters for each of these flows at the measurement station $2.8 \mathrm{~m}$ downstream of the plate's leading edge are in Table 1. The free stream Taylormicroscale Reynolds numbers, $R e_{\lambda 0}$, in these measurements ranged from 20 to 550, while the FST intensity at the measurement station ranged from $0.25 \%$ to $10.5 \%$. The boundary layer Reynolds number, $R e_{\theta}$, based on the momentum-thickness, $\theta$, varied from 550 to 2840 (Table 1).

Table 1: Parameters for the eight flows studied, including the free stream turbulent Reynolds number, $R e_{\lambda 0}$; the free stream mean streamwise velocity, $U_{0}$; the boundary layer thickness, $\delta$, based on $99.5 \%$ of the free stream velocity; the boundary layer momentum thickness, $\theta \equiv{ }_{0} \int^{\infty}\left(U / U_{0}\right)\left(1-\left(U / U_{0}\right) d y\right.$; the free stream turbulence intensity; the friction velocity, $u_{\tau}$; the Reynolds number based on momentum thickness, $R e_{\theta} \equiv U_{0} \theta / v$; the Reynolds number based on wall stress, $R e_{\tau} \equiv u_{\tau} \delta / v$; the ratio of the

free stream turbulent lengthscale to the boundary layer thickness, $L / \delta$, where $L$ is from the peak of the free stream energy spectrum; and the ratio of the longitudinal free stream velocity fluctuation r.m.s. to the friction velocity, $\left\langle u^{2}\right\rangle_{0}^{1 / 2} / u_{\tau}$.

\begin{tabular}{|c|c|c|c|c|c|c|c|c|c|}
\hline$R e_{\lambda 0}$ & $U_{0}\left(m s^{-1}\right)$ & $\delta(\mathrm{cm})$ & $\theta(\mathrm{cm})$ & $\left(\left\langle u^{2}\right\rangle^{1 / 2} / U\right)_{0}$ & $u_{\tau}\left(m s^{-1}\right)$ & $R e_{\theta}$ & $R e_{\tau}$ & $L / \delta$ & $\left\langle u^{2}\right\rangle_{0}^{1 / 2} / u_{\tau}$ \\
\hline 20 & 6.25 & 6.99 & 0.59 & $0.25 \%$ & 0.2671 & 2460 & 1245 & - & 0.059 \\
\hline 60 & 7.71 & 7.08 & 0.55 & $1.4 \%$ & 0.3293 & 2840 & 1560 & - & 0.315 \\
\hline 160 & 2.29 & 8.38 & 0.36 & $7.8 \%$ & 0.1211 & 550 & 680 & 2.8 & 1.480 \\
\hline 260 & 3.70 & 7.31 & 0.31 & $8 \%$ & 0.1878 & 775 & 915 & 4.4 & 1.557 \\
\hline 450 & 6.73 & 6.56 & 0.33 & $10.0 \%$ & 0.3145 & 1465 & 1375 & 5.5 & 2.173 \\
\hline 500 & 7.52 & 5.34 & 0.28 & $10.2 \%$ & 0.3523 & 1400 & 1250 & 7.0 & 2.131 \\
\hline 550 & 8.15 & 8.09 & 0.36 & $10.2 \%$ & 0.3747 & 1980 & 2020 & 5.2 & 2.256 \\
\hline 550 & 8.49 & 7.27 & 0.32 & $10.5 \%$ & 0.3963 & 1810 & 1920 & 5.1 & 2.229 \\
\hline
\end{tabular}


a)

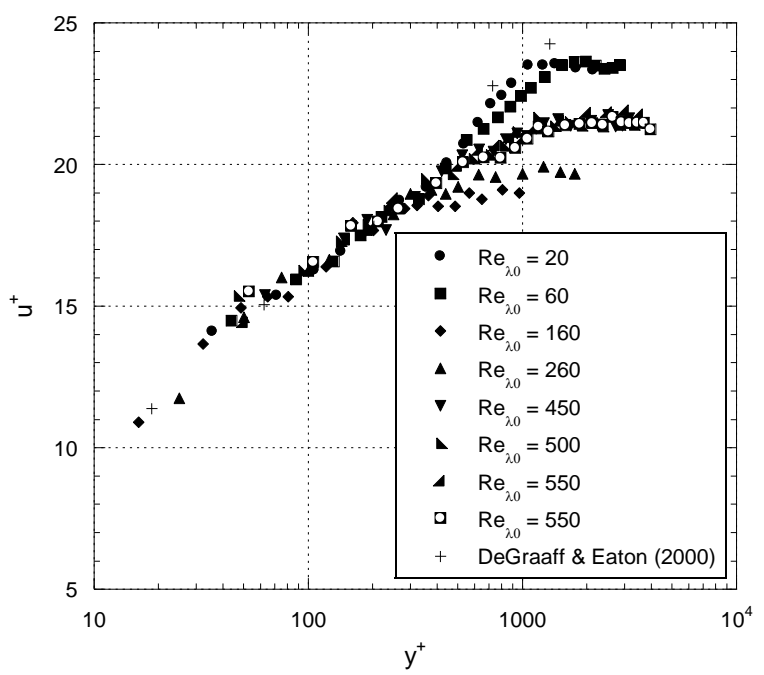

b)

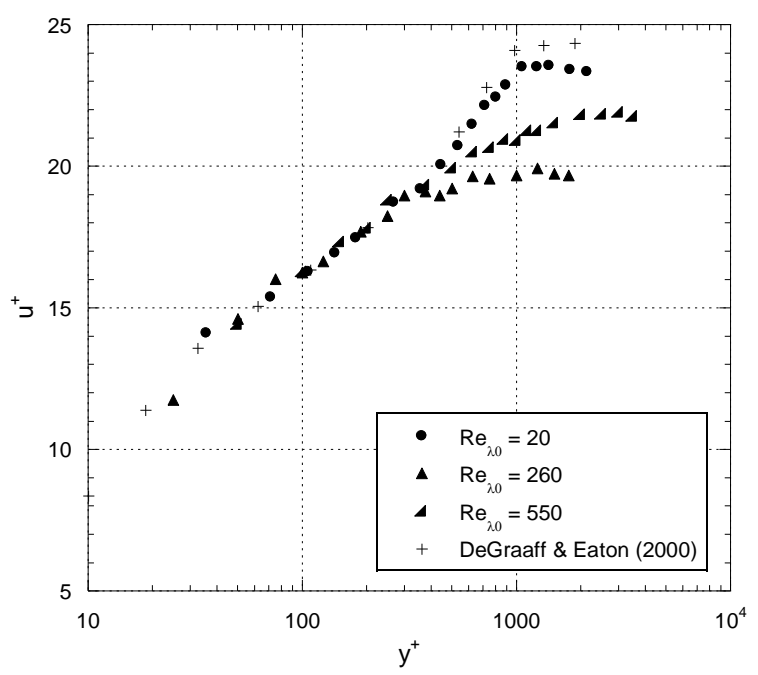

Figure 2: Mean velocity profiles normalized by inner variables for: a) all cases and b) example cases. Data for a boundary layer without FST from DeGraaff and Eaton (2000) are included for comparison [16].

Figure 2a shows the mean velocity profiles of all eight cases normalized by inner variables, $u_{\tau}$ and $v$. For turbulent boundary layers with little or no free stream turbulence, the near-wall velocity profile is assumed to follow the log-law of the wall:

$$
\frac{U}{u_{\tau}}=\frac{1}{\kappa} \ln \frac{y u_{\tau}}{v}+C
$$


where $\kappa$ is the von Karman constant with value $\kappa=0.41, C$ is a constant taken to be 5.0, and $u_{\tau}$ is the friction velocity. Thole and Bogard [10] showed that this law remains valid for determining wall shear stress even in flows with free stream turbulence intensities up to $20 \%$. In this experiment, a fit to the log-law determined each flow's friction velocity. Figure 2a also includes mean velocity data for a classical flat-plate turbulent boundary layer of $R e_{\theta}=2900$ [16]. All profiles display a log-region at least a decade wide with the cases with little FST displaying the closest match to the DeGraaff and Eaton data [16].

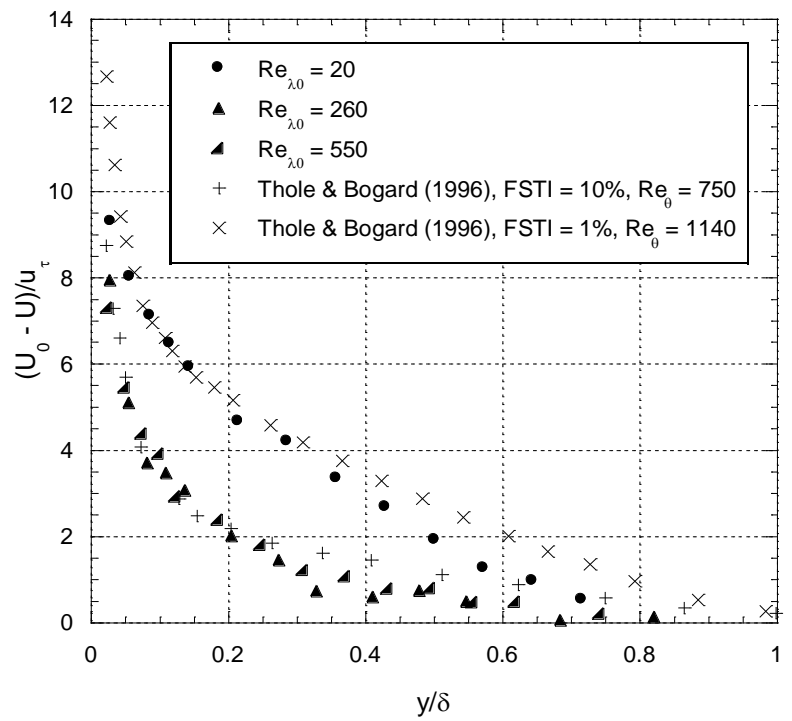

Figure 3: Mean velocity defect profiles with comparison to Thole and Bogard (1996) [10].

Increased levels of FST decrease the wake strength in the outer portion of the boundary layer, as observed by previous investigations $[7,10,13]$. The velocity defect profiles (Figure 3) show this strong effect. Included in Figure 3 are data from Thole and Bogard [10] with comparable free stream turbulence intensities to the present data. The agreement between the data sets is remarkably good given the very different methods of generating the free stream turbulence, although there is some 
disagreement in the outer portion of the boundary layer, possibly due to the lower $\operatorname{Re}_{\theta}$ values of the Thole and Bogard experiment [10]. The wake defect can be quantified by means of the wake parameter, $\Pi$, defined by:

$$
\frac{U}{u_{\tau}}=\frac{1}{\kappa} \ln \frac{\delta u_{\tau}}{v}+C+\frac{2 \Pi}{\kappa}
$$

For strong free FST this parameter becomes negative. To correlate mean flow parameters for turbulent boundary layers with free stream turbulence, Hancock and Bradshaw [6] defined an empirical parameter, $\beta$ :

$$
\beta=\frac{\frac{\left\langle u^{2}\right\rangle^{1 / 2}}{U}}{\frac{L_{e}^{u}}{\delta}+2}
$$

where the length-scale $L_{e}{ }^{u}$ is defined:

$$
L_{e}^{u}=-\frac{\left\langle u^{2}\right\rangle^{3 / 2}}{U_{0} \frac{d\left\langle u^{2}\right\rangle}{d x}}
$$

In the present work, the values of $\Pi$ and $\beta$ for the $R e_{\lambda 0}=260$ case were -0.395 and 1.465 , respectively. For the $R e_{\lambda 0}=550$ case, the values were -0.371 and 2.25 . These values are consistent with those observed by Thole and Bogard [10]. 


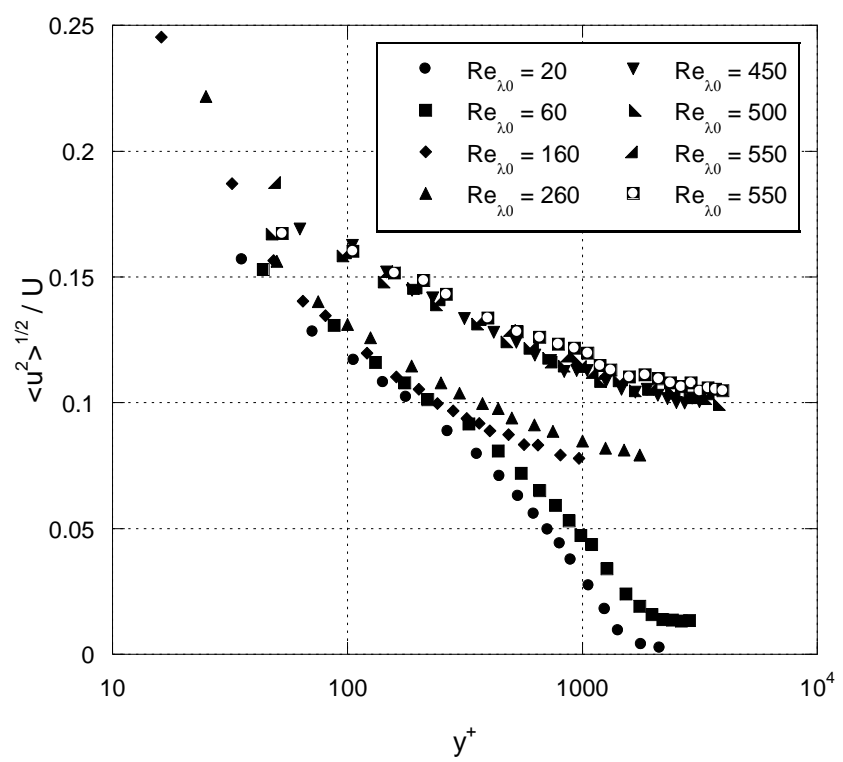

Figure 4: Turbulence intensity profiles.

Figure 4 shows the variation in turbulence intensity over the boundary layer. In both Figures 2 and 4, the current data divide into three basic categories: a boundary layer with very low free stream turbulence $\left(R e_{\lambda 0}=20,60\right)$; a boundary layer with high free stream turbulence $\left(\operatorname{Re}_{\lambda 0}=450-550\right)$ and a moderate boundary layer Reynolds number $\left(1465<R e_{\theta}<1980\right)$; and a boundary layer with significant free stream turbulence $\left(R e_{\lambda 0}=160,260\right)$ and a low boundary layer Reynolds number $\left(\operatorname{Re}_{\theta}=550,775\right)$. In Figure $2 \mathrm{a}$, the top branch corresponds to the first case, that of a developed boundary layer with little or no turbulence. We refer to this type as 'near-canonical.' The middle branch in Figure 2a belongs to the cases with developed boundary layers and high FST. Low $\operatorname{Re}_{\theta}$ boundary layers with significant FST ( $\sim 8 \%$ intensity) are represented in the lowest branch. In our results, we will focus on the three profiles shown in Figure 2b, as these exemplify the three basic categories. Other profiles will only be shown if needed for clarification. 
Figure 5: Normalized variances and covariances: a) $\left\langle u^{2}\right\rangle$, b) $\left\langle v^{2}\right\rangle$, and c) $\langle u v\rangle$. Data for a boundary layer without FST from DeGraaff and Eaton (2000) are included for comparison [16]. 
a)

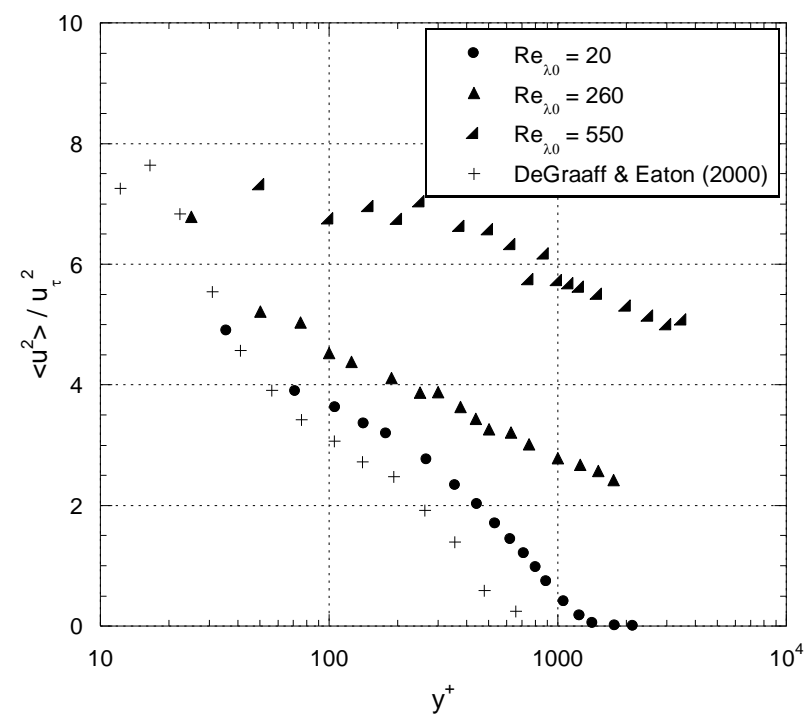

b)

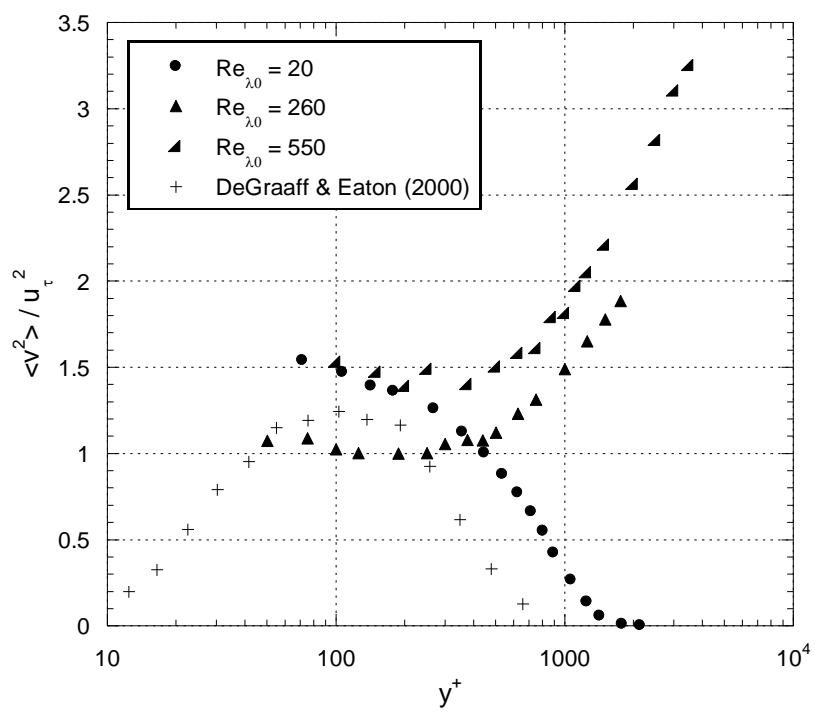


c)

Figure 5 (Continued)

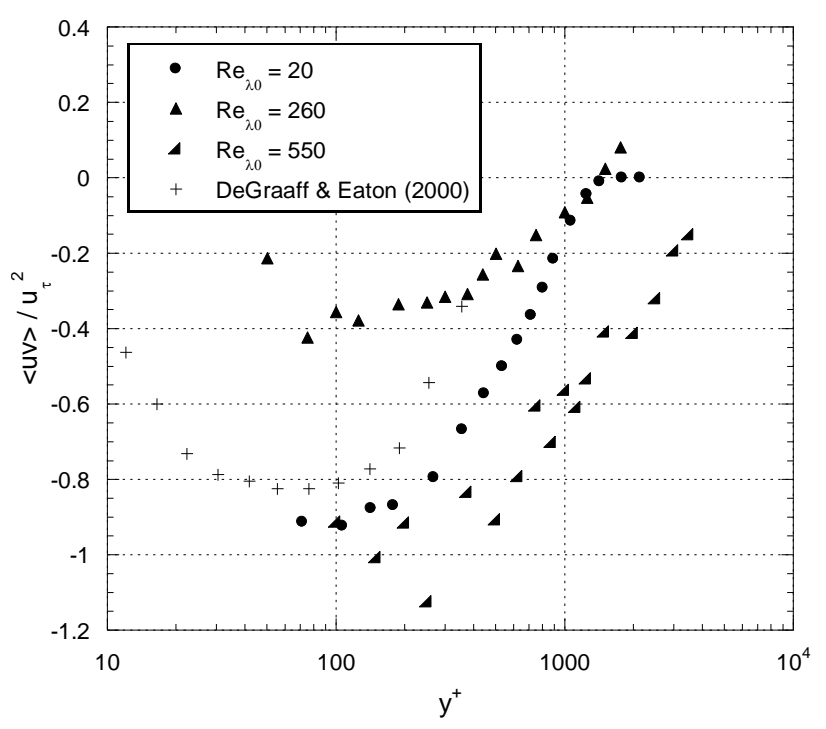

Figure 5 displays the normalized variances and covariances for the flow, including data from DeGraaff and Eaton's (2000) boundary layer experiment without free stream turbulence [16]. In the near-canonical case, the free stream variances are zero and increase inside the boundary layer, as in a classical boundary layer. For the cases with FST, the streamwise variance begins at a non-zero free stream value and increases in the boundary layer (Figure 5a). The plate-normal variance, $\left\langle v^{2}\right\rangle$, however, begins at a non-zero free stream value and decreases inside the boundary layer (Figure 5b). This trend for boundary layers with high FST has been reported by others $[10,12]$ and can be attributed to the influence of the wall on the vertical turbulent component as discussed in Hunt and Graham's (1978) study of free stream turbulence near planar boundaries [8]. They show that, in order to satisfy both the free stream conditions and the no-slip condition on the wall, continuity requires that $\left\langle v^{2}\right\rangle$ decrease from its free stream value inside the boundary layer. The covariance profiles (Figure 5c) show the highly turbulent case has stronger negative values than the near canonical case. The lower magnitude in the intermediate $\left(R e_{\lambda 0}=260\right)$ case may 
indicate a $\operatorname{Re}_{\theta}$ dependence. Although the FST is slightly lower for this case, the markedly lower $R e_{\theta}$ allows the FST to have a comparatively greater effect on the flow than in the $R e_{\lambda 0}=550$ case.

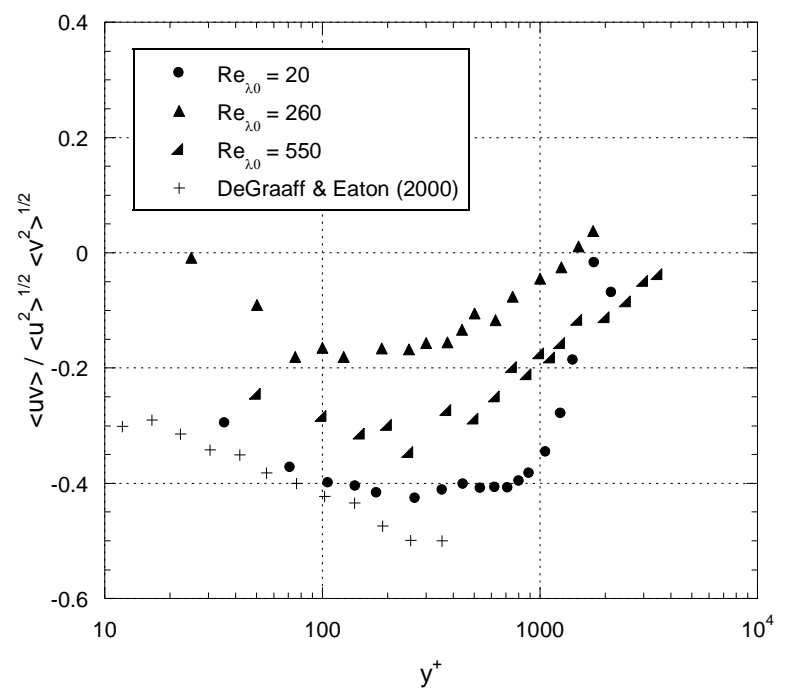

Figure 6: Cross-correlation coefficients profiles. Data for a boundary layer without FST from DeGraaff and Eaton (2000) are included for comparison [16].

Figure 6 shows the $u$-v cross-correlation coefficient measured throughout the boundary layers. For the near-canonical case, the correlation coefficient reaches a value of about -0.43 , consistent with other measurements and computations for a boundary layer without free stream turbulence [23]. The FST decreases the crosscorrelation coefficient throughout the boundary layer. Thole and Bogard [10] also report this trend. This result implies the penetration of uncorrelated $u$ - and $v$ components of the FST into the boundary layer fluid, thereby reducing the velocity correlation coefficient. The $\operatorname{Re}_{\lambda 0}=260$ case has the lowest cross correlation coefficient, consistent with Figure 5. 
a)

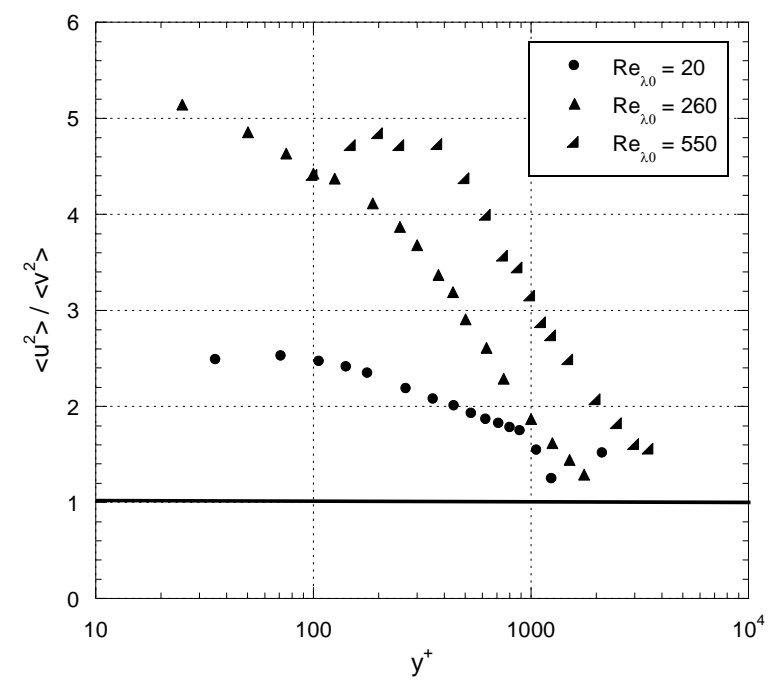

b)

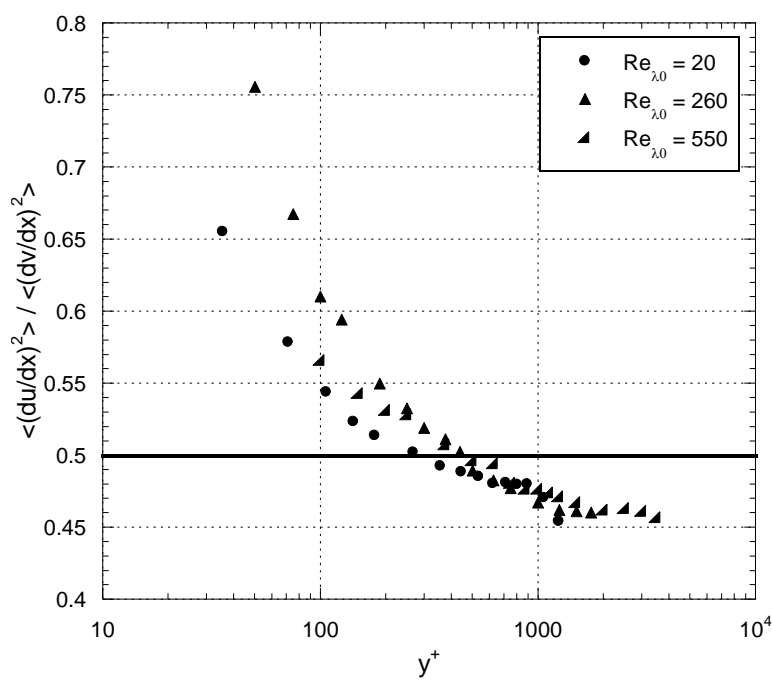

Figure 7: Profiles of anisotropy at: a) the large-scale and b) the small-scale.

The effect of free stream turbulence on the anisotropy, $\left\langle u^{2}\right\rangle /\left\langle v^{2}\right\rangle$, of the boundary layer is shown in Figure 7. Near the wall, the near-canonical boundary layer is increasingly anisotropic at the large-scale. With added FST, the boundary layer's anisotropy begins increasing at distances further from the wall and reaches greater 
Figure 8: Near-wall (open) and freestream (filled) $u$-direction velocity PDFs for: a) $R e_{\lambda 0}=20$, b) $R e_{\lambda 0}=260$, and c) $R e_{\lambda 0}=550$. Solid lines represent a Gaussian PDF with equivalent mean and r.m.s. velocities. 


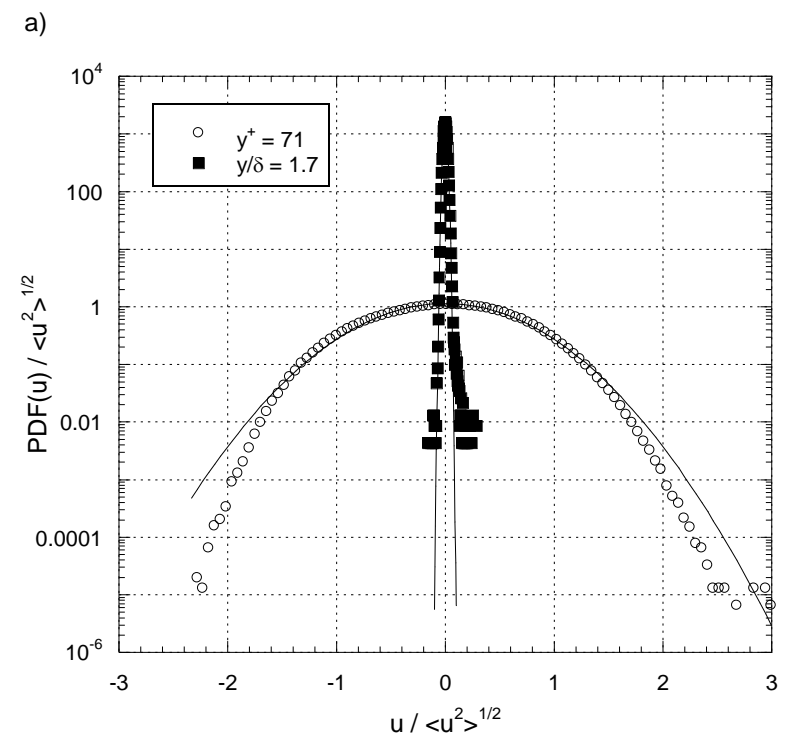

b)

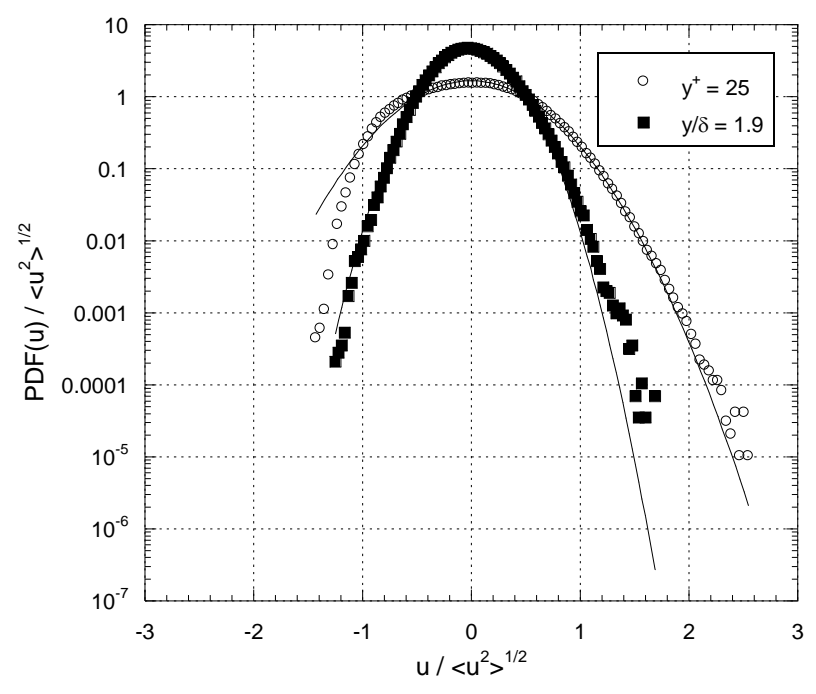


c)

Figure 8 (Continued)

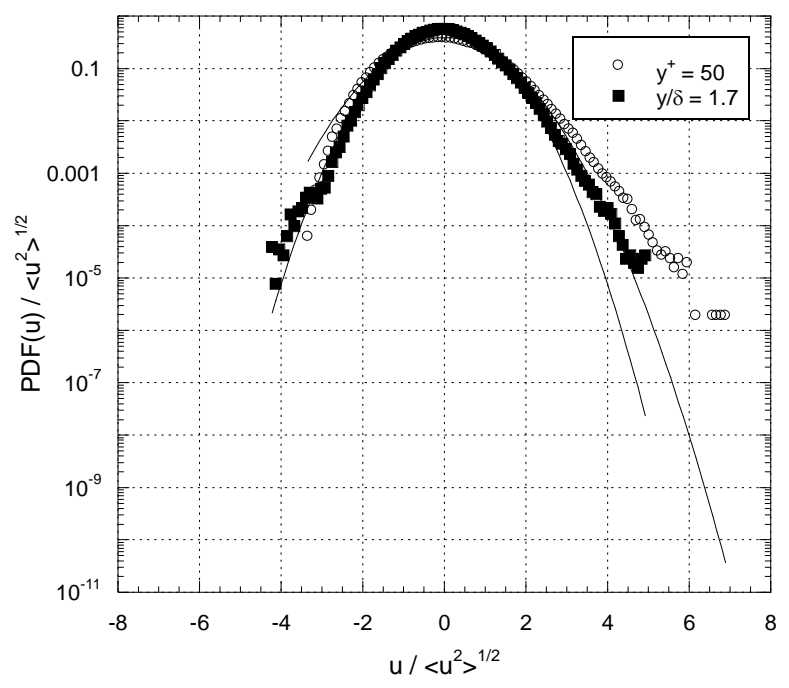

levels of anisotropy than in the near-canonical case. This trend is due to the stronger influence of the wall on fluctuations in the plate-normal direction. The ratio of the streamwise and plate-normal velocity derivatives, displayed in Figure 7b, shows increasing anisotropy of the small scales, but the degree to which the free stream affects the small-scales is less marked than its effect on the large scales. Notice that the FST has a small-scale (derivative) anisotropy of 0.45 , lower than the isotropic value of 0.5 . We cannot explain this departure.

Figure 8 shows normalized velocity probability distribution functions (PDFs) for these three flows at near-wall (open symbol) and free stream (filled symbol) distances. A Gaussian profile with equivalent mean and r.m.s. velocities is shown as a solid line for each; none of the profiles is strongly non-Gaussian. For the near-canonical case, the relative widths of the near-wall and free stream PDFs indicate a large difference in r.m.s. velocity, as was indicated by the trends in the streamwise velocity variance in Figure 5a. Both PDFs display a slight skewness toward the positive side. The cases 
with FST have PDFs with similar widths, indicating r.m.s. values that are closer in value in the near-wall and the free stream than for the near-canonical case. The PDFs also show a greater skewness toward the positive side at both the near-wall and free stream levels as the free stream turbulence is increased. These relative differences are more evident in Figure 9, which shows profiles of the $u$ and $v$ skewness. An additional data set, that of $\operatorname{Re}_{\lambda 0}=60$, has been included for clarity in Figures 9 (and in Figures 10, 11, and 12 to follow) because it shows a smoother transition in skewness and kurtosis values across the boundary layer. In Figure 9a, the skewness in the streamwise direction shows a sharp transition at the edge of the boundary layer where the near-laminar free stream meets the wall-generated turbulence. Figure $9 \mathrm{~b}$ indicates similar behavior in the plate-normal skewnesses. The signs of the skewnesses imply that, in the outer portion of a boundary layer with little FST, an upward motion is likely to be accompanied by a decrease in the streamwise motion. In contrast, the skewness profiles of the cases with FST show nearly constant values from the free stream to the wall, demonstrating the efficacy of the mixing in the outer portion of the boundary layer in both the streamwise and plate-normal directions. 
a)

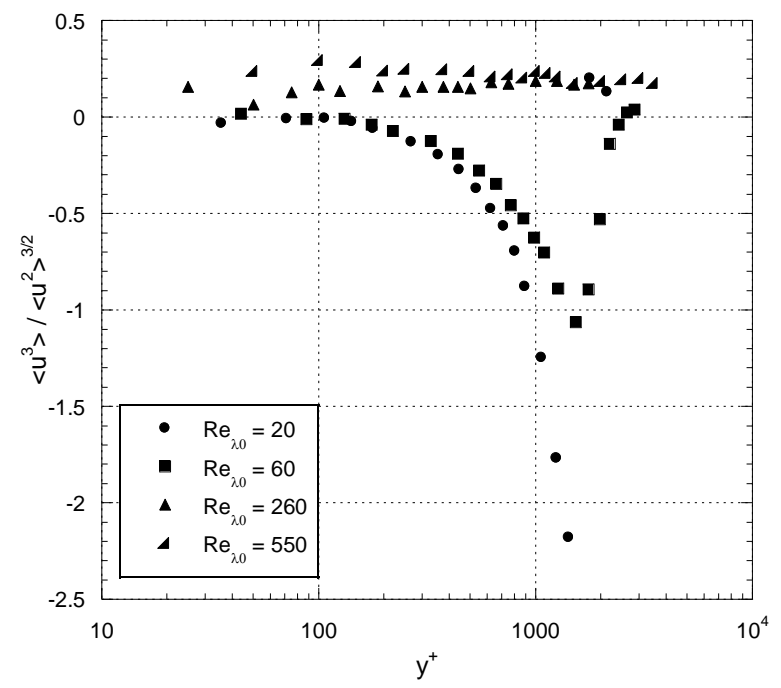

b)

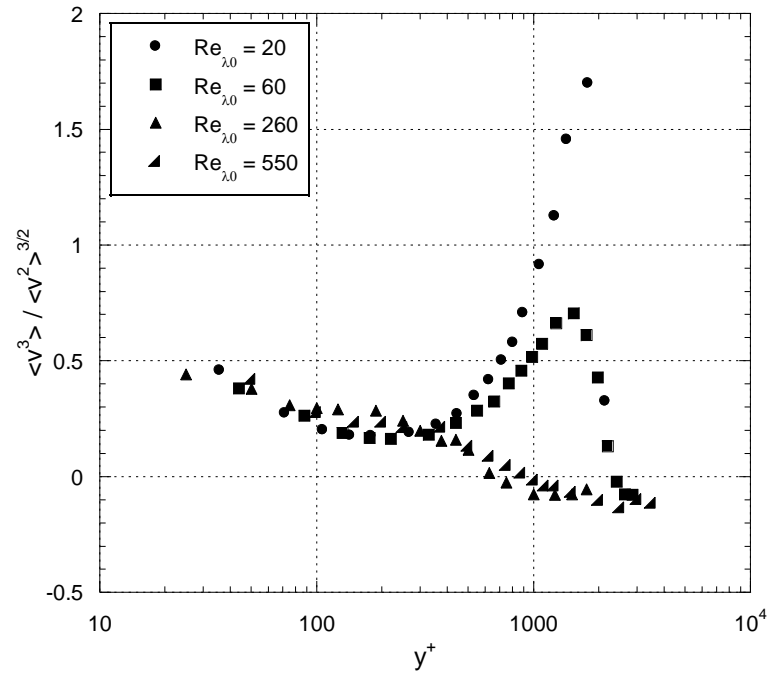

Figure 9: Large-scale skewness profiles in the: a) $u$-direction and b) $v$-direction.

As with the skewness, the kurtosis profiles in Figure 10 show that the presence of FST smears the effects of a transition between a turbulent boundary layer and a nearlaminar free stream. Note that the streamwise profiles of kurtosis are close to the Gaussian value of 3 throughout the boundary layer, with the exception of the transition region in the outer part of the near-canonical flow. The kurtosis profiles for the plate- 
normal direction show slightly more deviation from a value of 3 but still indicate essentially Gaussian behavior for much of the boundary layer, especially with FST.

a)

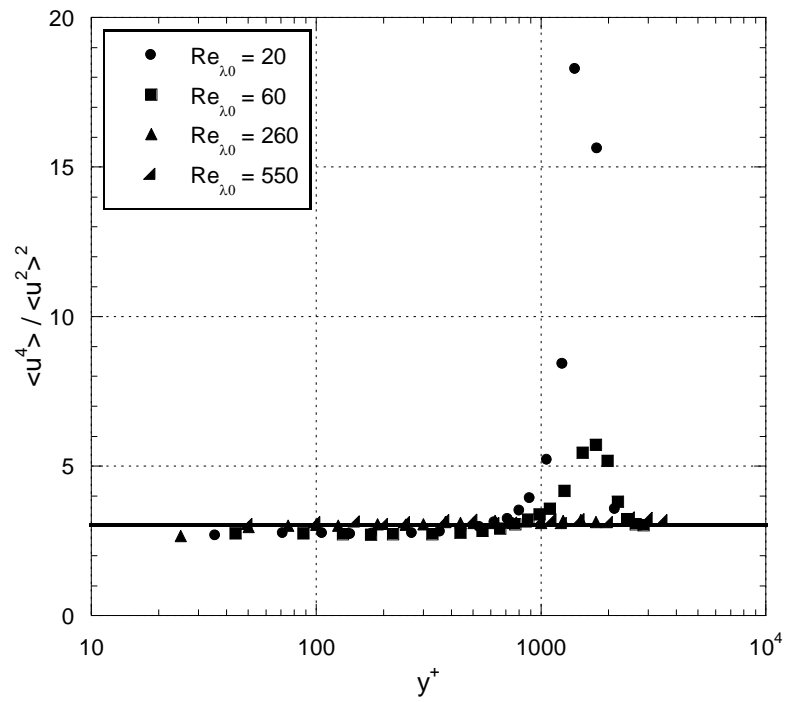

b)

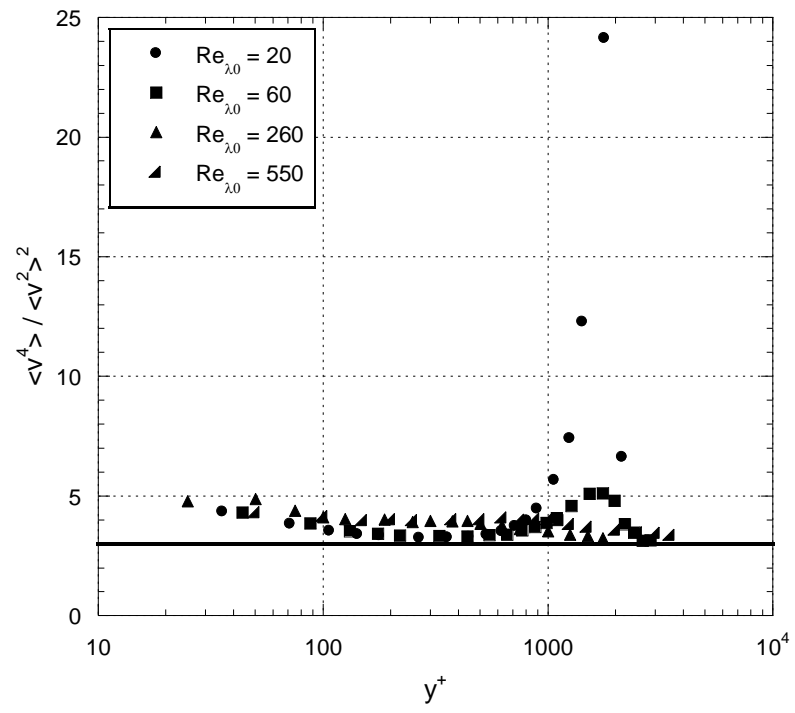

Figure 10: Large-scale kurtosis profiles in the: a) $u$-direction and b) $v$-direction. A Gaussian distribution has a kurtosis of 3 , shown here with a bold line. 
a)

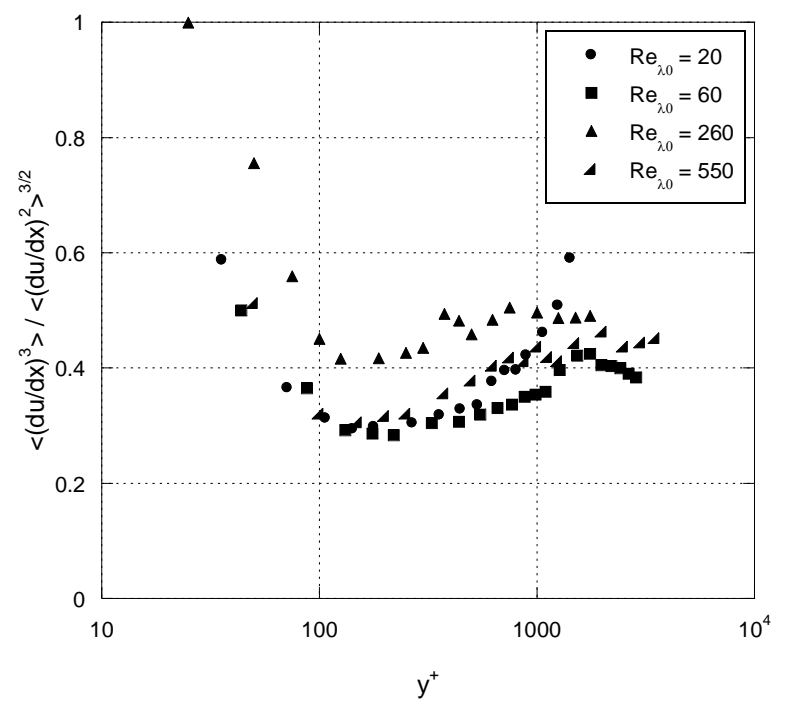

b)

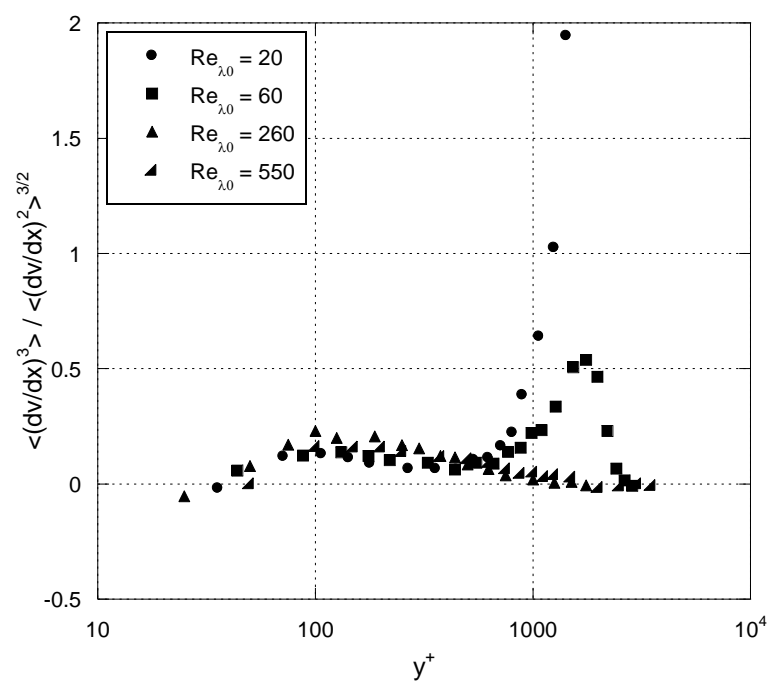

Figure 11: Small-scale (derivative) skewness profiles in the: a) $u$-direction and b) $v$ direction.

Small-scale (derivative) skewness values are shown in Figure 11. In the streamwise direction, the small-scale skewness values range between $0.3-0.5$ for much of the boundary layer, with and without FST (Figure 11a). The near-canonical case has stronger skewnesses on the outer edge of the boundary layer due to the interface between the nearly laminar free stream and the turbulent boundary layer. All cases 
a)

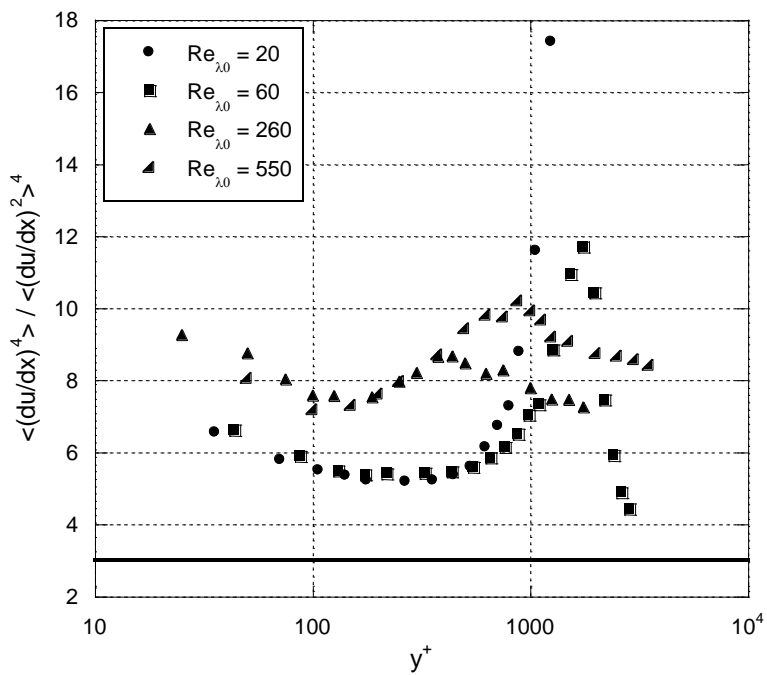

b)

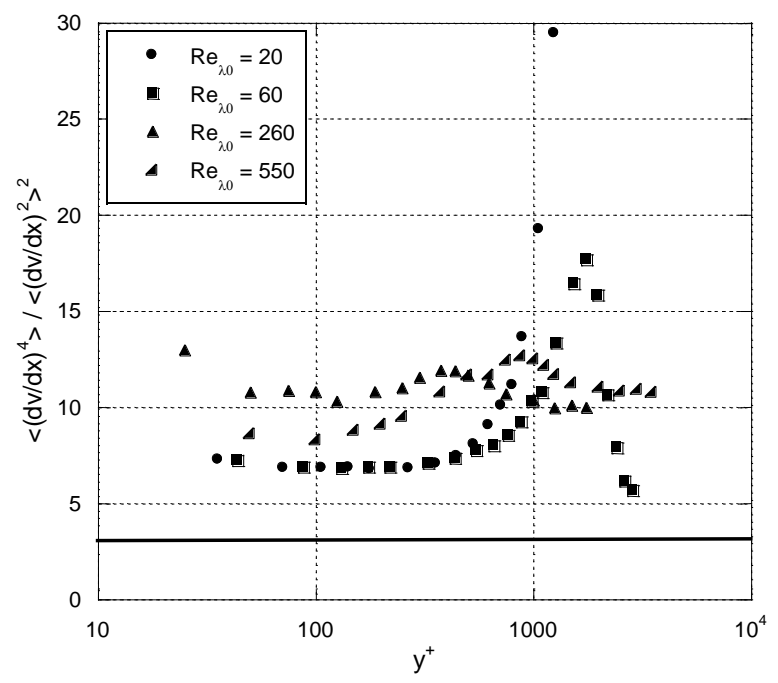

Figure 12: Small-scale (derivative) kurtosis profiles in the: a) $u$-direction and b) $v$ direction. A Gaussian distribution has a kurtosis of 3, shown here with a bold line.

show increasing skewness below $y^{+} \sim 100$, with a larger increase for the $\operatorname{Re}_{\lambda 0}=260$ case. There is greater collapse of the small-scale (derivative) skewnesses in the platenormal direction, shown in Figure 11b. Aside from strong skewness near the edge of the near-canonical boundary layers, there is very little skewness in the $v$-direction at the small scale. The small-scale (derivative) kurtosis values (Figure 12) show strongly non-Gaussian behavior in both the streamwise and plate-normal directions. The 
presence of FST decreases the kurtosis in the outer portion of the boundary layer, relative to the near-canonical cases, but the reverse is true closer to the wall. Thus the effects of the large-scale FST are evident at the small-scales deep into the boundary layer. The trends in the streamwise and plate-normal directions are the same, but the magnitudes of the plate-normal kurtosis values are greater.

We turn now to spectral results. Spectra were measured throughout the boundary layer for all eight cases. As before, however, we confine our reported results to our three example cases.

The evolution of the normalized power spectra, $E_{11}\left(\kappa_{l}\right)$, where $\kappa_{l}$ is the wavenumber in the streamwise direction, for the three flows is shown in Figure 13 with the spectra staggered from the bottom for clarity. The lowest curves in the figure are close to the wall and the top-most curves are in the free stream. The top spectrum in Figure 13a shows a narrow spectrum for the near-canonical case, indicative of very weak turbulence. The free stream spectra for the cases with FST, however, show the classic form with a relatively well-developed inertial subrange that increases with $R e_{\lambda 0}$. The Kolmogorov 1941 prediction with slope $-5 / 3$ is included for comparison [23]. Nearer to the wall these features disappear as anisotropy becomes more and more pronounced and the local $R e_{\lambda 0}$ decreases. In Figure 14, the same spectra are shown in a compensated form, which highlights the variations in the inertial subrange at different heights in the boundary layer. 


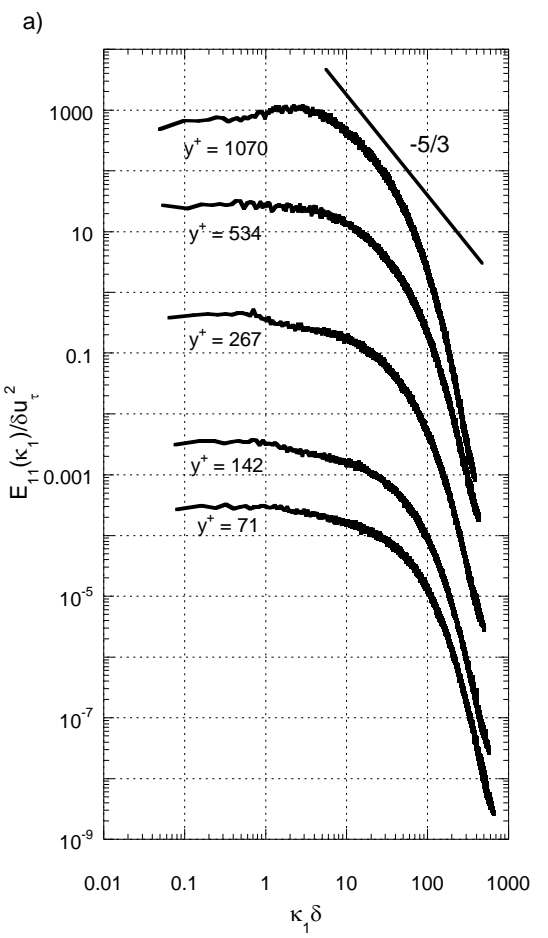

b)

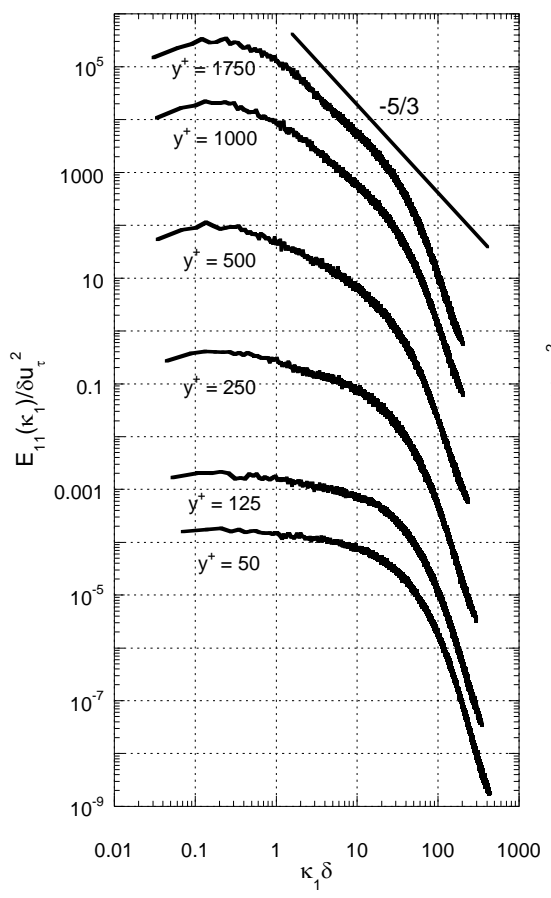

c)

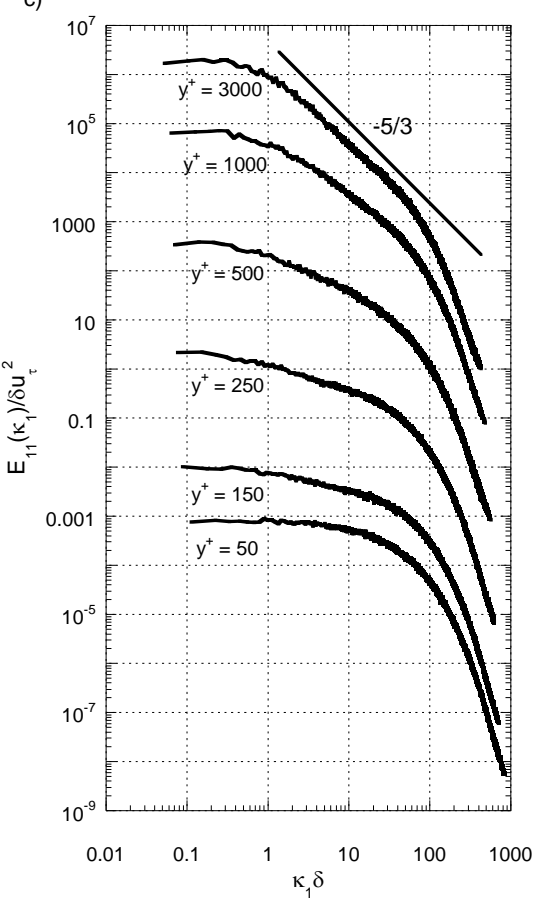

Figure 13: Evolution of boundary layer spectra for: a) $R e_{\lambda 0}=20$, b) $R e_{\lambda 0}=260$, and c) $R e_{\lambda 0}=550$. Spectra are staggered relative to the lowest spectrum. From bottom to top, the offset of each spectrum relative to the lowest is: $1,3,5,7$, and 8 decades. 
a)

b)

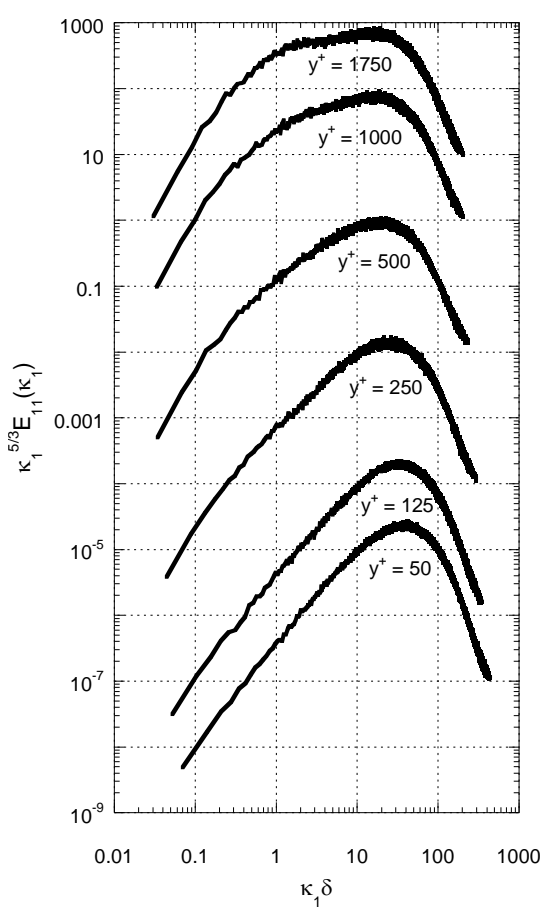

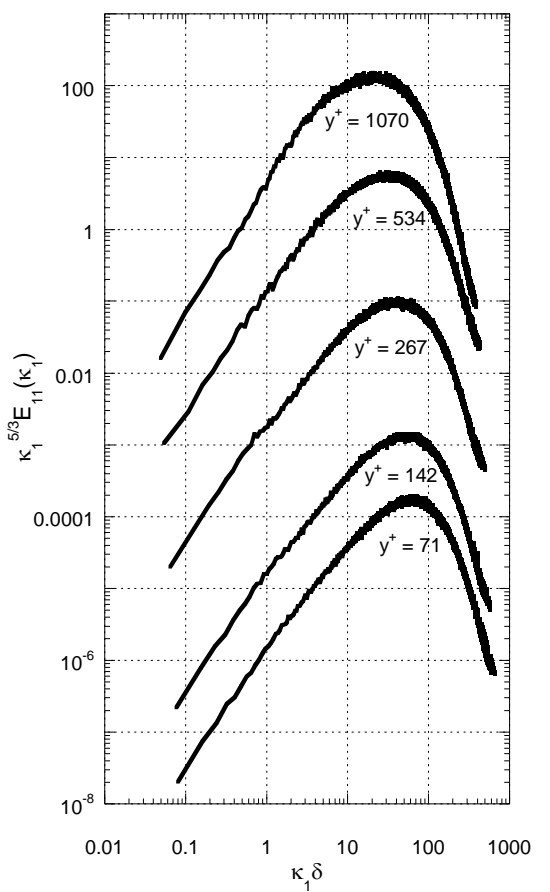

c)

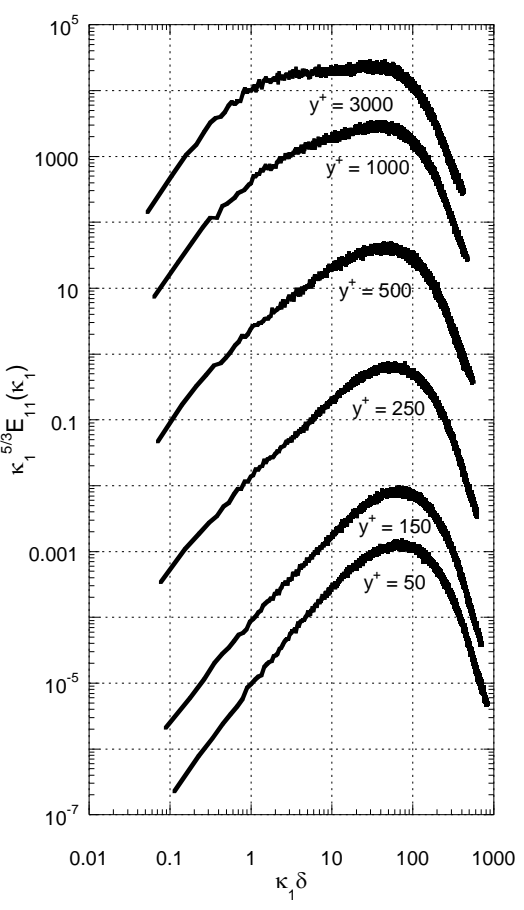

Figure 14: Evolution of compensated boundary layer spectra for: a) $R e_{\lambda 0}=20$,

b) $R e_{\lambda 0}=260$, and c) $R e_{\lambda 0}=550$. Spectra are staggered relative to the lowest

spectrum. From bottom to top, the offset of each spectrum relative to the lowest is: 1, $3,5,7$, and 8 decades. 
In order to emphasize the effects of the different length scales present in these flows, we focus on the energy spectrum, $\kappa_{1} E_{11}\left(\kappa_{1}\right)$. These spectra show how much energy there is at a given wavenumber at a particular location in the flow. By identifying lengthscales associated with the free stream and those associated with the boundary layer, one can compare their relative influence at different points in the boundary layer.

Hutchins and Marusic [15] studied the energy spectra of a classical boundary layer and identified two major lengthscales. Rather than using the wavenumber to describe these lengthscales, Hutchins and Marusic [15] refer to wavelengths, $\lambda\left(\equiv 2 \pi / \kappa_{l}\right)$; to facilitate comparison, we have kept this notation. In their experiments, the spectra peaked with a lengthscale of $\lambda^{+}\left(\equiv \lambda u_{\tau} / v\right)$ of approximately 1000 at $y^{+} \sim 15$. They associated this lengthscale with turbulent production near the wall. They also identified an outer lengthscale with size $\lambda \sim 6 \delta$. Hutchins and Marusic [15] posited that this outer lengthscale represented superstructures in the boundary layer that modulated near-wall production. They found that the outer scale became distinct at a height of $y / \delta \sim 0.06$. The two peaks drifted apart as the boundary layer Reynolds number increased. At high Reynolds numbers, the two peaks were not observed at the same height above the plate. For lower Reynolds number boundary layers (specifically $\operatorname{Re}_{\tau} \equiv u_{\tau} \delta / v \sim 1,000$ in the Hutchins and Marusic [15] experiment), the separation of the scales was reduced, resulting in a bimodal energy spectrum at $y / \delta \sim$ 0.06 , with both the inner and outer scales identifiable. 
Figure 15: Normalized near-wall energy spectra showing double peaks at $y / \delta \sim 0.05$ 0.06 for a) $R e_{\lambda 0}=20$, b) $R e_{\lambda 0}=260$, and c) $R e_{\lambda 0}=550$. 


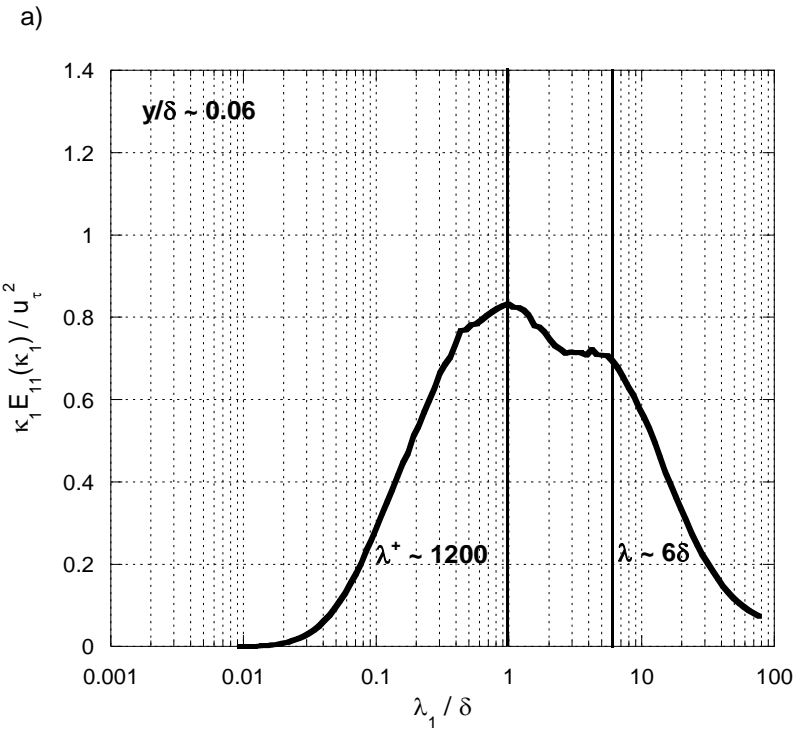

b)

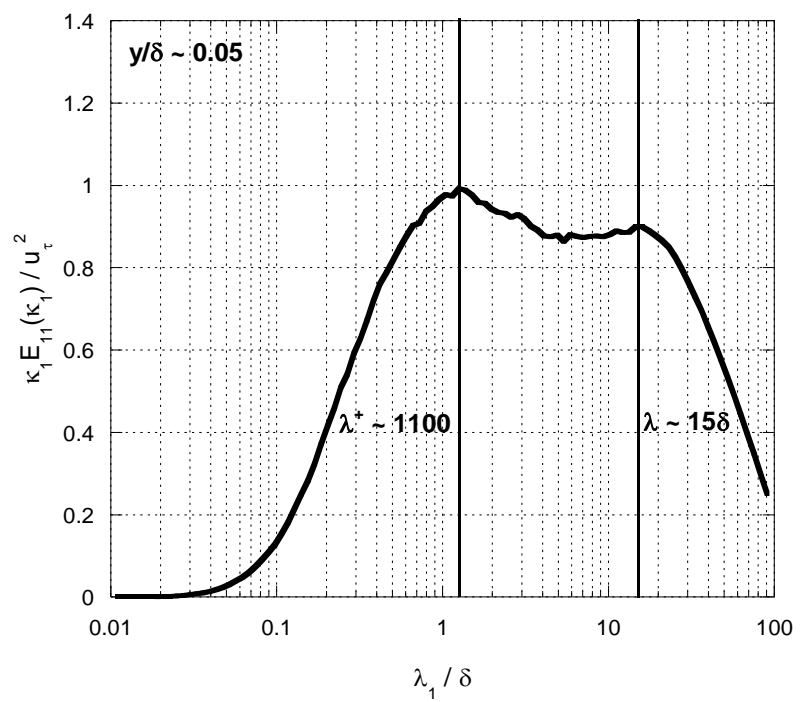


c)

Figure 15 (Continued)

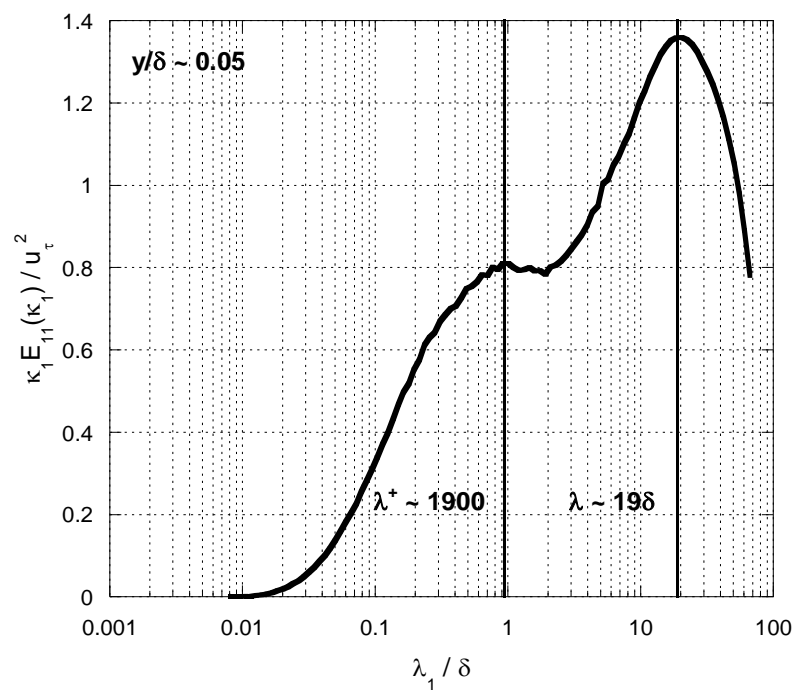

Figure 15 shows normalized energy spectra from the present experiment at $y / \delta \sim 0.05$ 0.06. The peaks in the spectra have been marked and labeled with their wavelengths. In Figure 15a, the near-canonical boundary layer's energy spectrum includes two peaks: an inner peak at $\lambda^{+} \sim 1200$ and an outer peak at $\lambda \sim 6 \delta$, in agreement with Hutchins and Marusic's (2007) findings [15]. With the addition of FST, one might expect the energy spectrum to contain three distinct peaks - two associated with the boundary layer and one associated with the free stream-but this behavior is not observed. Figure 15b, like Figure 15a, contains a double-peaked spectrum despite the addition of free stream turbulence. The relative locations of the peaks are shifted, however, with the peaks for the $\operatorname{Re}_{\lambda 0}=260$ case occurring at $\lambda^{+} \sim 1100$ and $\lambda \sim 15 \delta$. While the location of the inner peak has not shifted significantly, the change in the outer peak from $\lambda \sim 6 \delta$ to $\lambda \sim 15 \delta$ is substantial. This larger wavelength is associated with the FST, which, in this experiment, is approximately five times larger than the boundary layer depth (see Table 1). Figure 15c displays the energy spectrum for the highly turbulent free stream case and shows behavior very similar to the intermediate 
case. In this case, the double peaks are shifted rightward with the inner, boundarylayer peak occurring at $\lambda^{+} \sim 1900$ and the outer, free stream-associated peak occurring at $\lambda \sim 19 \delta$. Note the very high energy in the free stream compared to that at the inner lengthscale. Thole and Bogard [10] also noted a double peak in their spectrum for a FST of $12.5 \%$, but its evolution was not studied.

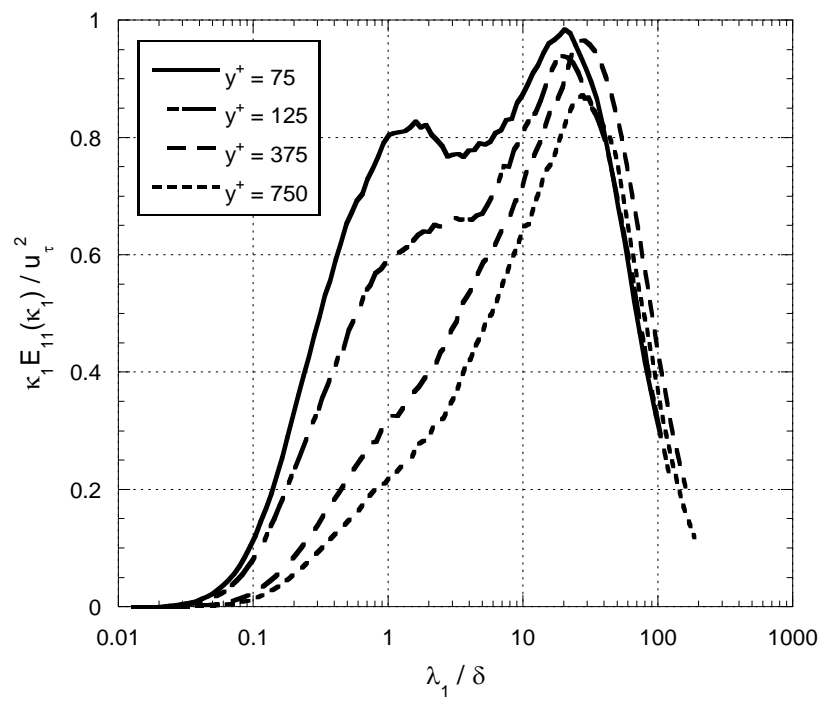

Figure 16: Evolution of normalized energy spectra from the wall to the free stream for $R e_{\lambda 0}=260$.

The evolution of the energy spectra at different heights in the flow is shown in Figure 16 for the intermediate $R e_{\lambda 0}=260$ case. Near the wall, the $y^{+}=75$ curve has two distinct peaks; in contrast, the free stream spectrum, taken at $y^{+}=750$, contains only a single peak. At intermediate heights in the flow, the inner peak disappears, indicating its association with the boundary layer, while the outer peak, which must be associated with the free stream turbulence, remains nearly unchanged. 
Figure 17: Three-dimensional (top) and contour plots (bottom) of energy spectra throughout the boundary layer: a) and b) $R e_{\lambda 0}=20$; c) and d) $R e_{\lambda 0}=260$; and e) and f) $R e_{\lambda 0}=550$. 


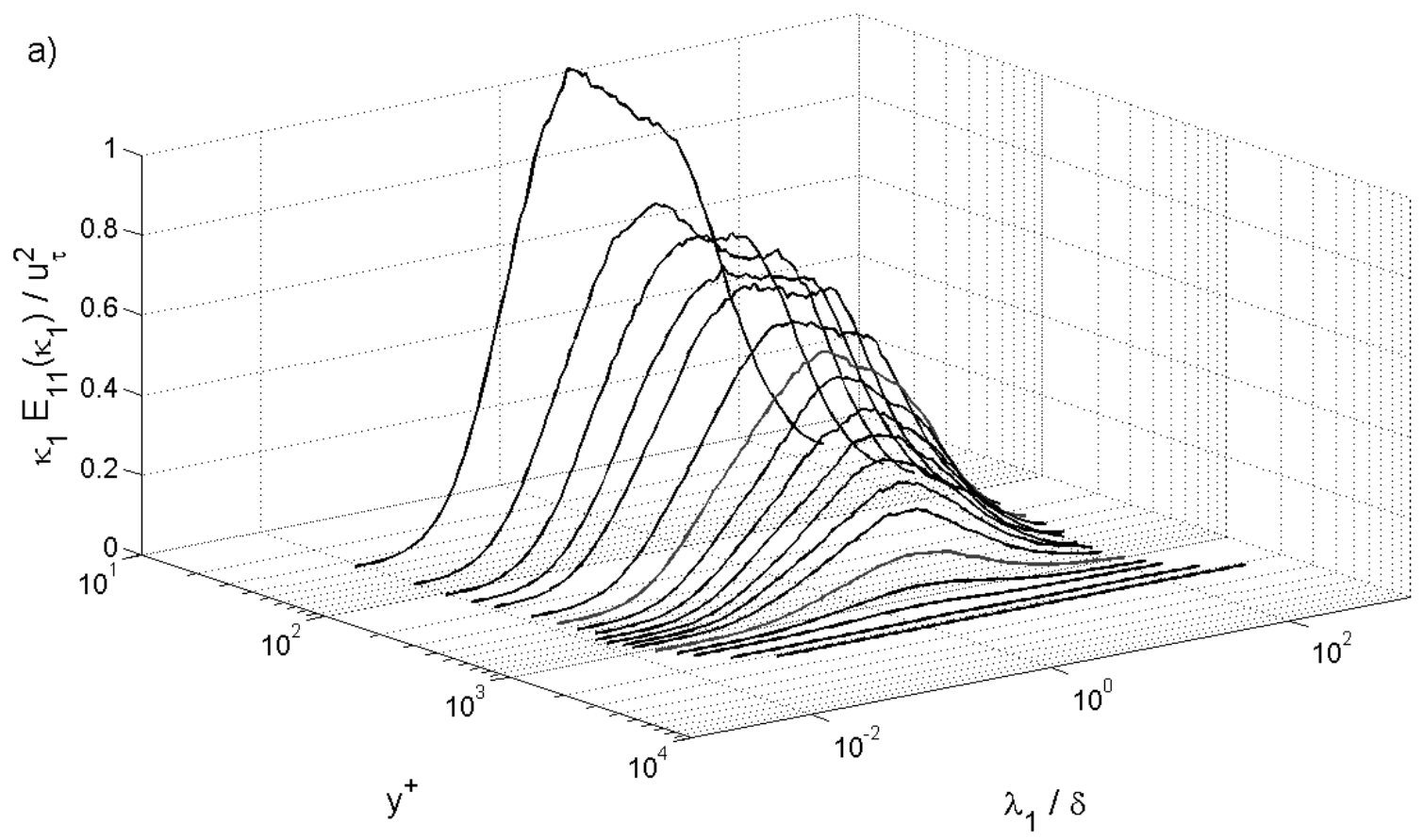

b)

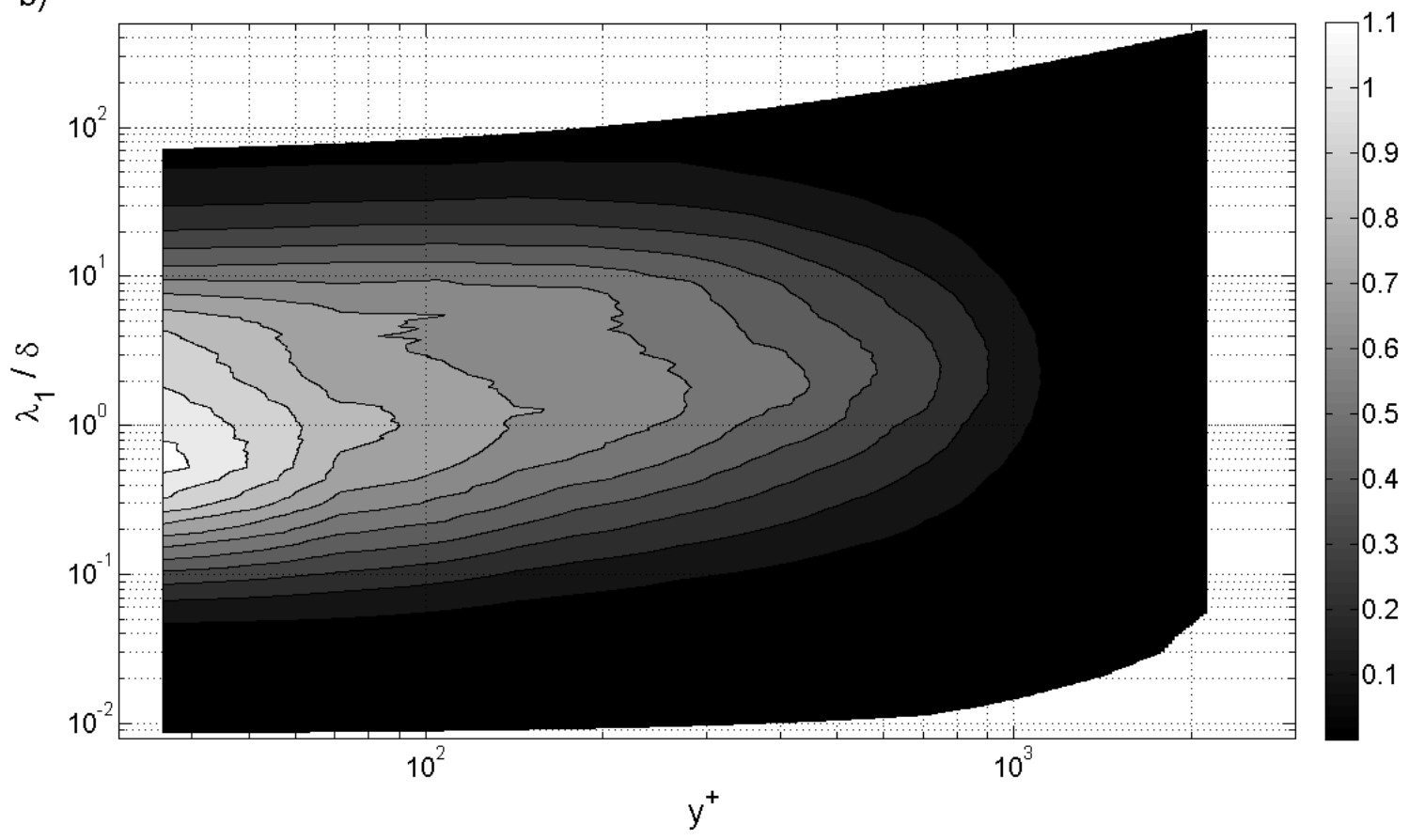


Figure 17 (Continued)

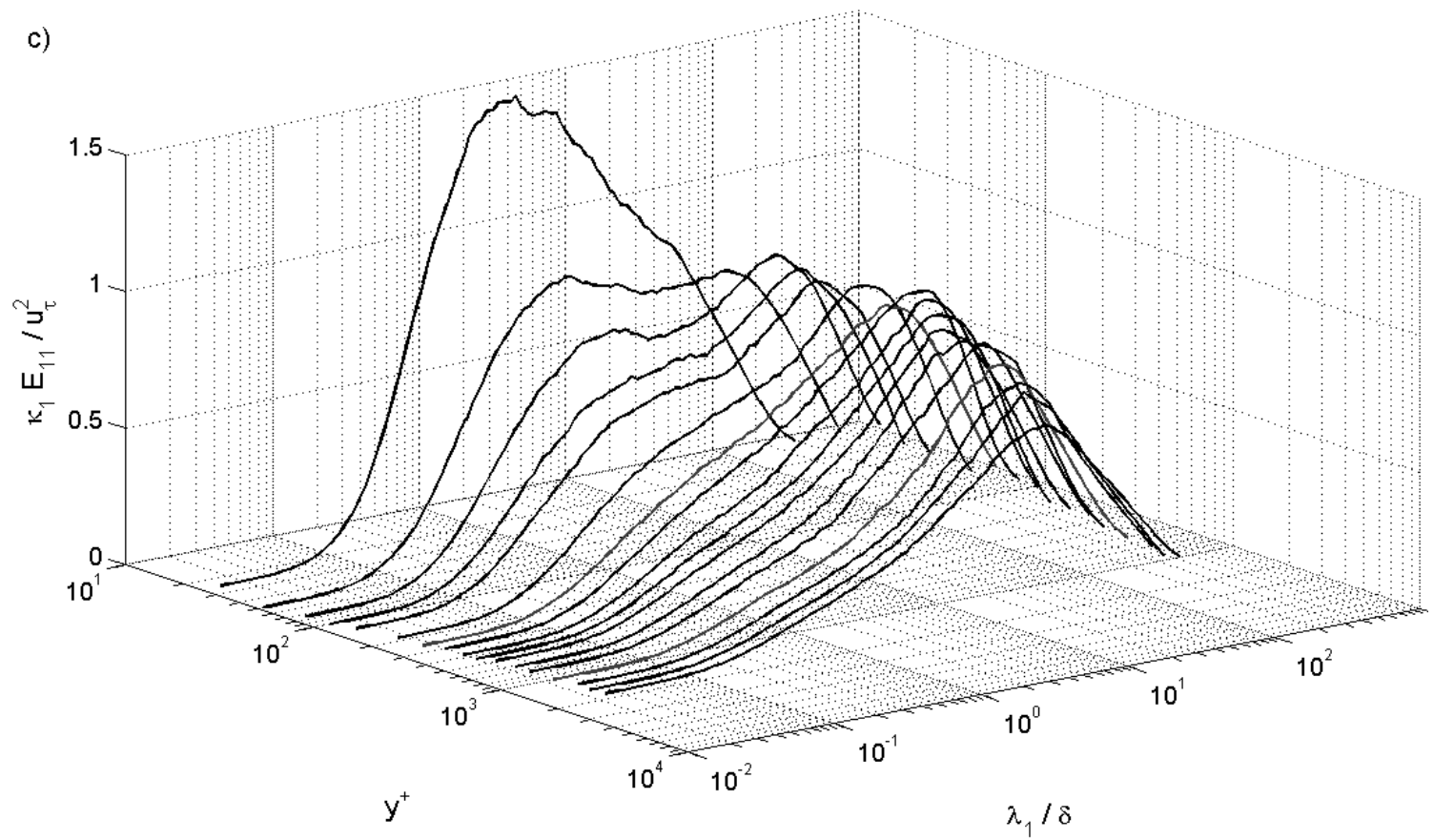

d)

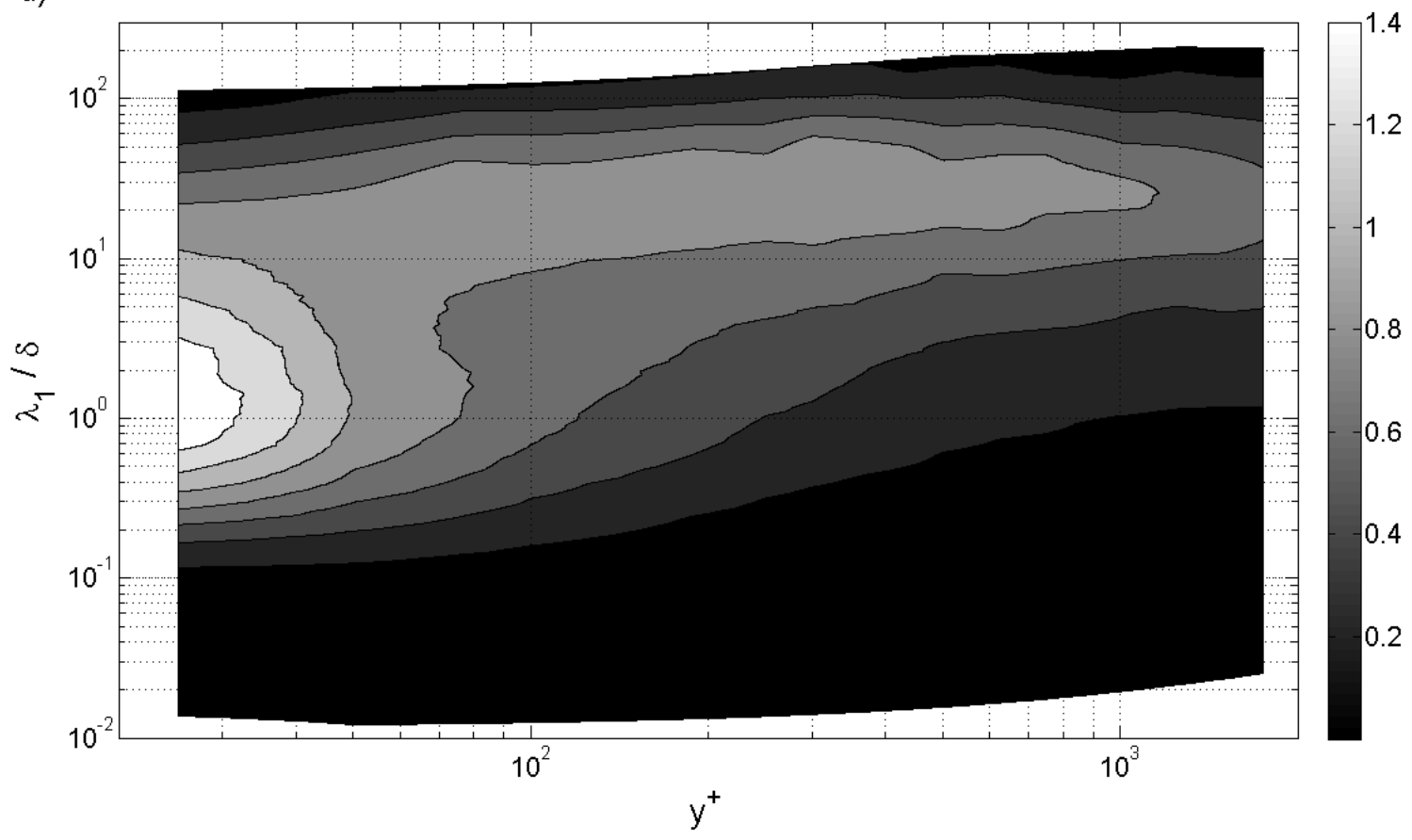


Figure 17 (Continued)

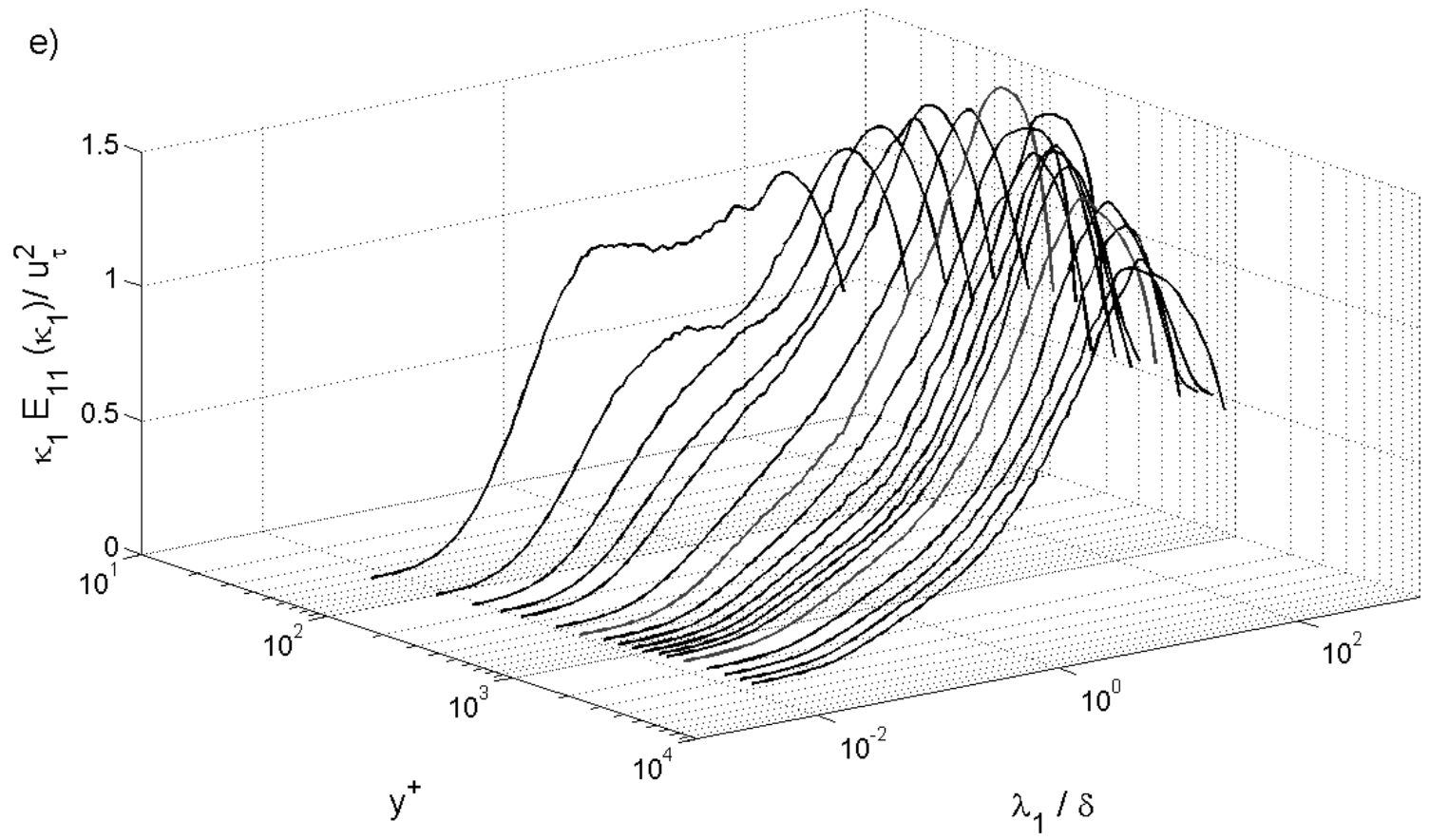

f)

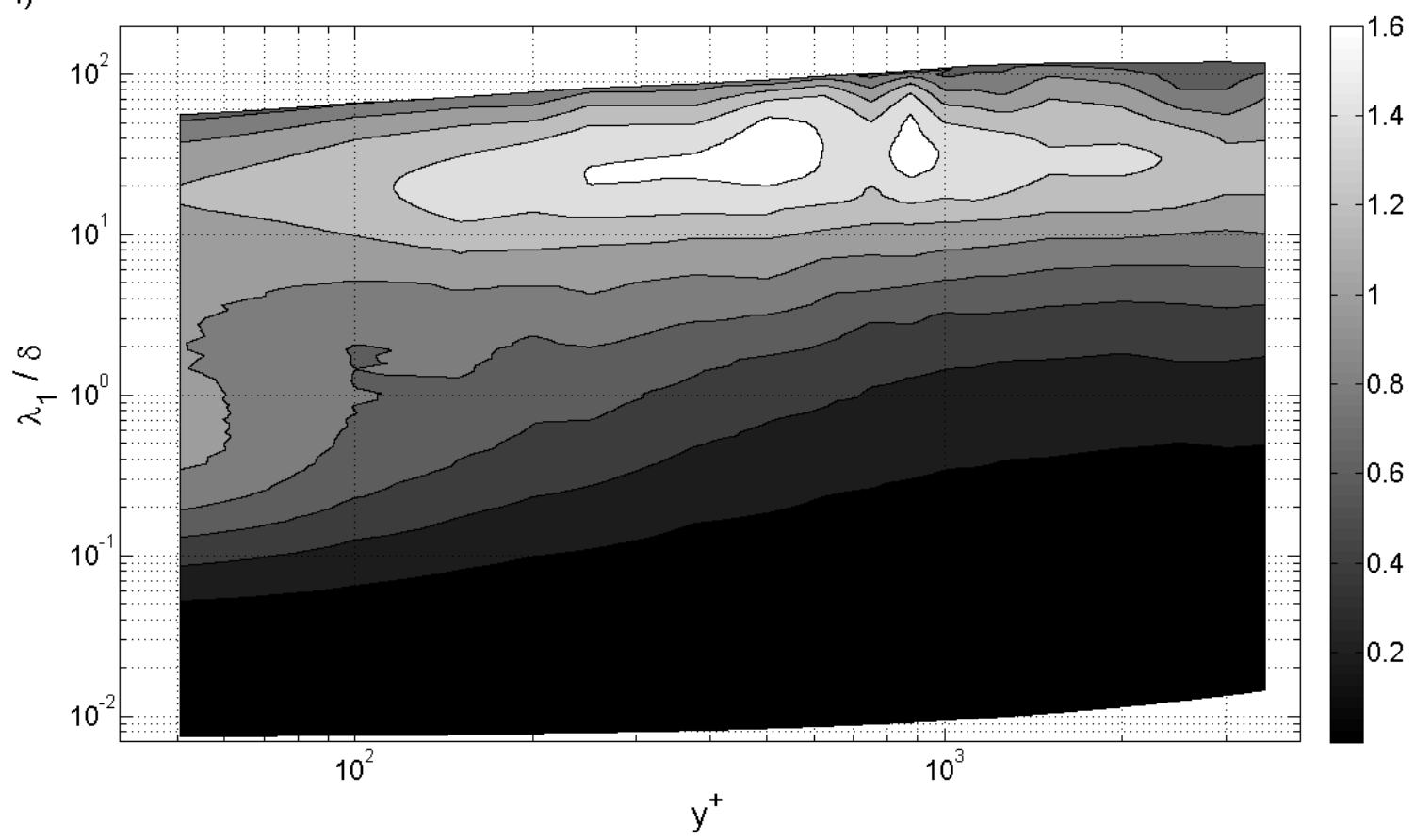


Following Hutchins and Marusic [15], maps of the full evolution of these spectra over the height of the boundary layer for all three cases are displayed in Figure 17. Here the maps on the left show the energy spectra at different heights in the boundary layer as a three-dimensional graph. To the right, the same data are given in the form of a contour plot, in essence showing the three-dimensional graphs as viewed from above.

Figures $17 \mathrm{a}$ and $17 \mathrm{~b}$ show the evolution of the near-canonical boundary layer. As expected, the spectrum grows from zero in the free stream to the double-peaked spectrum reported by Hutchins and Marusic [15] midway through the boundary layer. Further into the boundary layer, the inner peak begins to dominate the spectrum. Unlike the near-canonical case, the $R e_{\lambda 0}=260$ case, shown in Figures $17 \mathrm{c}$ and $17 \mathrm{~d}$, has a single distinct peak in the free stream, and this peak retains its magnitude until the inner boundary layer peak develops and takes precedence at approximately $y^{+} \sim$ 40. A comparison of Figures $17 \mathrm{~b}$ and $17 \mathrm{~d}$ indicates just how substantial the effect of FST is on the structure throughout the boundary layer. Similarly, the highly turbulent free stream case shown in Figures $17 \mathrm{e}$ and $17 \mathrm{f}$ contains a prominent free stream peak until $y^{+} \sim 70$ with an inner boundary layer peak developing midway through the boundary layer. As with the previous case, the differences in boundary layer structure relative to the near-canonical boundary layer's evolution are striking. 


\section{CONCLUSIONS}

We have described the effects of FST on the structure of a turbulent boundary layer. The conditions of the FST varied in $R e_{\lambda 0}$ from 20 to 550 and in intensity from $0.25 \%$ to $10.5 \%$. The degree of development in the boundary layer also varied, from $\operatorname{Re}_{\theta}$ of 550 to 2840 . Results fell into three categories: a boundary layer with very low free stream turbulence $\left(R e_{\lambda 0}=20,60\right)$; a boundary layer with high free stream turbulence $\left(R e_{\lambda 0}=450-550\right)$ and a moderate boundary layer Reynolds number $\left(1465<R e_{\theta}<\right.$ $1980)$; and a boundary layer with significant free stream turbulence $\left(\operatorname{Re}_{\lambda 0}=160,260\right)$ and a low boundary layer Reynolds number $\left(\operatorname{Re}_{\theta}=550,775\right)$.

In addition to presenting mean flow, variance, and cross-correlation statistics, which were in agreement with previous investigations $[6,7,10]$, we presented higher order moments for the large- and small-scale (derivative) statistics throughout the boundary layer. At the large-scale, FST reduced the skewness across the boundary layer, especially in the outer region, compared to a boundary layer with little FST (Figure 9). The small-scale (derivative) skewnesses in the plate-normal direction (Figure 11b) were similarly reduced by FST in the outer region of the boundary layer. The derivative skewness in the streamwise direction (Figure 11a) was less affected by FST but showed an increase close to the wall for the $R e_{\lambda 0}=260$ case. The kurtosis profiles (Figures 10 and 12) showed that the effects of FST vary in different ways for the large- and small-scales of the boundary layer. At the large-scale (Figure 10), FST promotes Gaussian behavior throughout the boundary layer and greatly reduces the large-scale intermittency of the flow. In contrast, FST caused an increase in nonGaussian behavior at the small-scales inside the boundary layer (Figure 12), implying that the large-scale FST affects the small-scales. 
Spectral analysis provided further evidence of the impact of free stream turbulence on the boundary layer. Hutchins and Marusic [15] found a double-peaked energy spectrum for a boundary layer without FST. They argued that the large-scale motions represented by the spectrum's outer peak had a modulating effect on the smaller scales of the boundary layer. They observed that the large-scale superstructures "appear to actively modulate the production of near-wall scales, and the extent to which it does so will increase with the Reynolds number (as the outer peak becomes increasingly comparable in energy to the inner peak)" [15]. In the current work, we observed that a double-peak also occurs in the energy spectrum for a boundary layer with FST. Moreover, the addition of FST above a boundary layer caused the energy in the outer peak of the energy spectrum to reach comparable or even greater levels than that of the inner peak (Figure 15b,c) even at a relatively low $R e_{\theta}$. The implication of this behavior is that the FST can have a substantial impact on the small-scales of the boundary layer, even at low boundary layer Reynolds numbers, as was seen with the derivative kurtosis (Figure 12).

Three-dimensional mapping of the energy spectrum over the boundary layer provided a visual representation of the impacts of FST on boundary layer structure (Figure 17). Energy from the free stream penetrated to depths below $y^{+}=100$. This result implies that large-scale FST is not only capable of affecting the smaller scales of the boundary layer, but that free stream energy is reaching deep into the boundary layer, allowing it to directly affect the inner scales of the boundary layer.

The effects observed here between large-scale free stream turbulence and the boundary layer at both the large- and small-scale have substantial implications, particularly for industrial flows. In situations where active control over turbulence is 
desirable (e.g. for mixing, heat exchange, drag reduction, etc.), large-scale FST has the potential to provide control over boundary layer scales well inside the boundary layer, even for relatively underdeveloped boundary layers. 


\section{REFERENCES}

[1] A. J. Thorpe and T. H. Guymer, "The nocturnal jet," Quarterly Journal of the Royal Meteorological Society, 103, 633 (1977).

[2] X. Wu, R. G. Jacobs, J. C. R. Hunt, and P.A. Durbin, "Simulation of boundary layer transition induced by periodically passing wakes," J. Fluid Mech. 398, 109 (1999).

[3] J. F. Manwell, J. G. McGowan, and A. L. Rogers, Wind energy explained (John Wiley and Sons, Ltd., Chichester, England, U.K., 2002).

[4] J. C. R. Hunt, P.A. Durbin, and X. Wu, "Interactions between freestream turbulence and boundary layers," (Center for Turbulence Research, Ann. Research Briefs, Stanford University, 1998).

[5] I. P. Castro, "Effects of free stream turbulence on low Reynolds number boundary layers," J. Fluids Engi. 106, 298 (1984).

[6] P.E. Hancock and P. Bradshaw, "The effect of free-stream turbulence on turbulent boundary layers," J. Fluids Engi. 105, 284 (1983).

[7] P.E. Hancock and P. Bradshaw, "Turbulence structure of a boundary layer beneath a turbulent free stream," J. Fluid Mech. 205, 45 (1989).

[8] J. C. R. Hunt and J. M. R. Graham, "Free-stream turbulence near plane turbulence," J. Fluid Mech. 84, 209 (1978).

[9] G. Charnay, J. Mathieu, and G. Comte-Bellot, "Response of a turbulent boundary layer to random fluctuation in the external stream," Phys. Fluids 19, 1261 (1976).

[10] K. A. Thole and D. G. Bogard, "High freestream turbulence effects on turbulent boundary layers," J. Fluids Engi. 118, 276 (1996).

[11] P. R. Bandyopadhyay, "Reynolds number dependence of the freestream turbulence effects on turbulent boundary layers," AIAA J. 30, 1910 (1992).

[12] D. M. Bott and P. Bradshaw, "Effect of high free-stream turbulence on boundary-layer skin friction and heat transfer," (Report No. MD-75, Dept. of Mech. Eng., Stanford University, 1997).

[13] M. F. Blair, "Influence of free-stream turbulence on turbulent boundary layer heat transfer and mean profile development, part I-experimental data," $J$. Heat Transfer. 105, 33 (1983). 
[14] S. Torres-Nieves, B. Brzek, J. Lebron, L. Castello, and C. Meneveau, “Addition of isotropic free-stream turbulence promotes anisotropy in a turbulent boundary layer," Bulletin of the APS, 53, 220 (2008).

[15] N. Hutchins and I. Marusic, "Large-scale influences in near-wall turbulence," Phil. Trans. Royal Soc. 365, 647 (2007).

[16] D. B. DeGraaff and J. K. Eaton, "Reynolds-number scaling of the flat-plate turbulent boundary layer," J. Fluid Mech. 422, 319 (2000).

[17] H. Makita, "Realization of a large-scale turbulence field in a small wind tunnel.," Fluid Dyn. Res. 8, 53 (1991).

[18] L. Mydlarski and Z. Warhaft, "On the onset of high-Reynolds-number gridgenerated wind tunnel turbulence," J. Fluid Mech. 320, 331 (1996).

[19] H. Kang, S. Chester, and C. Meneveau, "Decaying turbulence in an active-gridgenerated flow and comparisons with large-eddy simulation," J. Fluid Mech. 480, 129 (2003).

[20] Q. A. Ahmad, R. E. Luxton, and R. A. Antonia, "Characteristics of a turbulent boundary layer with an external turbulent uniform shear flow," J. Fluid Mech. 77, 369 (1976).

[21] S. Gerashchenko, N. S. Sharp, S. Neuscamman, and Z. Warhaft, "Lagrangian measurements of inertial particle accelerations in a turbulent boundary layer," $J$. Fluid Mech. 617, 255 (2008).

[22] K. Yoon and Z. Warhaft, "The evolution of grid-generated turbulence under conditions of stable thermal stratification," J. Fluid Mech. 215, 601 (1989).

[23] S. B. Pope, Turbulent Flows (Cambridge University Press, Cambridge, 2000). 